\title{
Sandro Fehr
}

\section{Energie für den Krieg. Schweizer Unternehmen als Zulieferer und Produzenten in der deutschen Stickstoff- wirtschaft während des Ersten Weltkriegs}

\begin{abstract}
The supply of the dual-use resource nitrogen became an existential problem for Germany during World War I. This article discusses how the German war economy attempted to solve the supply problem and how successful it was in doing so. The supply of nitrogen from their neutral neighbour Switzerland is discussed in order to show that Swiss companies produced nitrogen compounds in Switzerland itself, while at the same time also exporting intermediate products and resources to Germany for the manufacture of nitrogen compounds. Swiss companies also built factories on German soil that were part of the German nitrogen industry and were in some cases even operated with Swiss resources and labour.
\end{abstract}

JEL-Codes: N 44, N 53, N 54, N 74

Keywords: Kriegswirtschaft, Erster Weltkrieg, Rohstoffe, Rohstoffbewirtschaftung, Landwirtschaft, Salpeter, Ammoniak, Stickstoff, Energie, Deutschland, Schweiz

DOI 10.1515/jbwg-2015-0019

Die Mittelmächte sahen sich im Ersten Weltkrieg mit dem Problem konfrontiert, dass die Entente sie mit der Seeblockade und weiteren wirtschaftlichen Kampfmaßnahmen weitgehend vom Welthandel abschnitt. ${ }^{1}$ Für die deutsche Kriegswirtschaft erwies sich dabei insbesondere der Umstand als kritisch, dass sie von der Einfuhr einer ganzen Reihe kriegswichtiger Rohstoffe abhängig war, die nicht

1 W. Michalka, Kriegsrohstoffbewirtschaftung, Walter Rathenau und die „kommende Wirtschaft“, in: Ders. (Hg.), Der Erste Weltkrieg. Wirkung, Wahrnehmung, Analyse, München 1994, S. 485; G. Hardach, Der Erste Weltkrieg, 1914-1918, München 1973, S. 19.

Sandro Fehr (Dr.), Universität Bern, Historisches Institut, Abteilung für Wirtschafts-, Sozial- und Umweltgeschichte, Länggassstrasse 49, CH-3012 Bern, E-mail: sandro_fehr@icloud.com 
mehr oder nur noch in unzureichenden Mengen importiert werden konnten. $\mathrm{Zu}$ diesen zählte neben Metallerzen, Schwefel oder Rohöl auch Chilesalpeter, der als eine der wichtigsten Quellen gebundenen Stickstoffs sowohl für die landwirtschaftliche Düngung bzw. die Ernährung der Bevölkerung, als auch für die Herstellung von Explosivstoffen bzw. die Kriegführung unentbehrlich war. ${ }^{2}$

Im vorliegenden Artikel wird zunächst aufgezeigt, dass die Versorgung mit der Schlüsselressource Stickstoff bereits vor dem Ersten Weltkrieg ein international vielbeachtetes Thema war, das sich in Deutschland im Kontext des industriell geführten Kriegs aber schnell zu einem Problem von existenzieller Tragweite entwickelte. Im zweiten Kapitel wird zusammenfassend dargestellt, wie und mit welchem Erfolg die deutsche Kriegswirtschaft das Versorgungsproblem während des Ersten Weltkriegs zu lösen versuchte. Danach wird auf die Versorgungslage im neutralen Nachbarstaat Schweiz eingegangen, um anschließend die Rolle schweizerischer Unternehmen in der deutschen Stickstoffwirtschaft zu behandeln. Untersucht werden dabei sowohl Lieferungen von in der Schweiz hergestellten Stickstoffverbindungen, als auch von Zwischenerzeugnissen und Ressourcen, die in Deutschland zur Gewinnung von Stickstoffverbindungen verwendet wurden. In einem weiteren Kapitel wird aufgearbeitet, wie Schweizer Unternehmen in Deutschland selbst tätig und somit Teil der deutschen Kriegswirtschaft wurden. Abgeschlossen wird der Artikel mit einem kurzen Fazit.

\section{Relevanz und Gewinnung der Schlüsselressource Stickstoff}

Die Relevanz der Ressource Stickstoff ergibt sich zunächst einmal daraus, dass sie ein wichtiger Nährstoff für das Pflanzenwachstum ist. Gemäß dem bereits im 19. Jahrhundert von Justus von Liebig (1803-1873) entdeckten „Gesetz des Minimums“ kann ein Mangel an Stickstoff im Boden auch nicht durch die Zufuhr anderer Nährstoffe, wie Kalium oder Phosphor, kompensiert werden. ${ }^{3}$ Während Pflanzen in der Natur durch den Stickstoffkreislauf meist ausreichend mit gebundenem Stickstoff versorgt sind, muss in der Landwirtschaft ab einer gewissen Intensität der Bodennutzung hoffremder Stickstoffdünger bzw. Handelsdünger

2 S. Fehr, Die „Stickstofffrage“ in der deutschen Kriegswirtschaft des Ersten Weltkriegs und die Rolle der neutralen Schweiz, Nordhausen 2009, S. 10-11.

3 V. Smil, Enriching the Earth. Fritz Haber, Carl Bosch, and the Transformation of World Food Production, Cambridge, Massachusetts 2001, S. 7. 
zugeführt werden. Ansonsten droht eine Verarmung des Bodens. Gerade in dichtbesiedelten, industrialisierten Staaten wie Deutschland reichte daher die landwirtschaftliche Nutzfläche ohne zusätzliche hoffremde Düngung nicht aus, um die Ernährung der Bevölkerung sicherzustellen. Der „wichtigste Stickstoffdünger“, der dabei um die Wende zum 20. Jahrhundert zur Anwendung kam, war der in Südamerika abgebaute Chilesalpeter. ${ }^{4}$

Die Abhängigkeit von dieser aus Übersee eingeschifften Ressource wurde von Wissenschaftlern bereits lange vor Beginn des Ersten Weltkriegs international als problematisch beurteilt. Der prominenteste unter ihnen war der britische Chemiker William Crookes (1832-1919), der 1898 in einem Referat davor warnte, dass die Weltbevölkerung aufgrund ihres starken Wachstums und der grundsätzlich beschränkten Anbaufläche schon bald nicht mehr ernährt werden könne: „England and all civilised nations stand in deadly peril of not having enough to eat. As mouths multiply, food resources dwindle. " ${ }^{5}$ Eine Hungersnot lasse sich nur durch eine Steigerung der landwirtschaftlichen Flächenerträge vermeiden, wozu große Mengen von Stickstoffdünger bzw. Chilesalpeter erforderlich seien. Crookes prognostizierte jedoch, dass die Bestände von Chilesalpeter bereits in 20 bis 30 Jahren erschöpft sein würden. ${ }^{6}$ Rettung versprach er sich aus dem Chemielabor: „It is the chemist who must come to the rescue of the threatened communities. It is through the laboratory that starvation may ultimately be turned into plenty. " $\mathrm{Er}$ glaubte, dass die Abhängigkeit von Chilesalpeter mit der industriellen Fixierung des in der Luft enthaltenen Stickstoffs durchbrochen werden könne. ${ }^{8}$

Crookes Prognose über den Zeitpunkt der Erschöpfung des Chilesalpeters wurde in der Fachwelt überwiegend als unkorrekt erachtet. Geteilt wurde hingegen seine Sorge über die Abhängigkeit von dieser Ressource sowie die Ansicht, dass sie mit der industriellen Stickstofffixierung überwunden werden könne. ${ }^{9} \mathrm{Zu}$ den frühesten Verfahren, mit denen der Stickstoff der Luft chemisch gebunden wurde, gehört das Lichtbogenverfahren. Dabei werden im Prinzip die in der Natur

4 H. Ost, Lehrbuch der technischen Chemie. Mit einem Schlussabschnitt „Metallurgie“, Hannover 1898, S. 145-146, 152; Fehr, Stickstofffrage, S. 26-31.

5 W. Crookes, The Wheat Problem. Based on Remarks Made in the Presidential Address to the British Association at Bristol in 1898, London 1899, S. 3.

6 Ebda., S. 42-43.

7 Ebda., S. 3.

8 Ebda., S. 46-47.

9 W. Rabius, Kritische Betrachtungen zur voraussichtlichen Lösung der Stickstofffrage, Jena 1907, S. 2-6; N. Caro, Die Stickstofffrage in Deutschland. Vortrag, gehalten am 23. April $1907 \mathrm{im}$ polytechnischen Verein zu München, Berlin 1908, S. 9, 23-26, 41; R. Huber, Zur Stickstoff-Frage. Eine Studie, Bern 1908, S. 32-36; H. Grossmann, Die Stickstofffrage und ihre Bedeutung für die deutsche Volkswirtschaft, Berlin 1911, S. 7-14. 
auftretenden Blitze, die im Stickstoffkreislauf für einen Teil der natürlichen Stickstoffbindung verantwortlich sind, im Labor imitiert. Wie der Name des Verfahrens schon sagt, kommt anstelle von Blitzen ein kontinuierlicher, elektrischer Lichtbogen zur Anwendung, welcher den in der Luft enthaltenen Stickstoff mit dem ebenfalls in der Luft enthaltenen Sauerstoff zur Reaktion bringt, wobei Stickoxide entstehen. Diese können mit Wasser zu Salpetersäure und diese beispielsweise mit Kalk zum Stickstoffdünger Calciumnitrat umgesetzt werden. Die Übertragung des Lichtbogenverfahrens vom Labormaßstab in den industriellen Maßstab erfolgte in den Jahren 1903-1907. ${ }^{10}$ Als nachteilig erwies sich bei diesem Verfahren der außerordentlich hohe Energieverbrauch. Die entsprechenden Fabriken wurden daher mehrheitlich in Ländern wie Norwegen, Schweden und der Schweiz gebaut, wo große Mengen vergleichsweise günstiger Wasserkraft verfügbar waren. ${ }^{11}$

Parallel zum Lichtbogenverfahren wurde zu Beginn des 20. Jahrhunderts auch das Kalkstickstoffverfahren zur industriellen Reife gebracht. Dieses besteht darin, Stickstoff mit Calciumcarbid zur Reaktion zu bringen, wobei Calciumcyanamid entsteht, das auch als Kalkstickstoff bezeichnet wird. Diese Stickstoffverbindung kann entweder direkt als Dünger eingesetzt oder mit Wasser zu Ammoniak weiterverarbeitet werden. Während der Produktionsschritt der Azotierung, d.h. der Reaktion des Karbids mit Stickstoff, lediglich eine Initialzündung erfordert und dann exotherm, d.h. unter Abgabe von Energie, verläuft, ist die Herstellung des Zwischenzeugnisses Calciumcarbid aus Kohle und Kalk im elektrischen Ofen sehr energieaufwendig. ${ }^{12}$ Insgesamt erforderte das Kalkstickstoffverfahren pro Einheit gebundenen Stickstoffs dennoch nur rund halb so viel Energie wie das Lichtbogenverfahren. ${ }^{13}$ Nachdem in Italien die erste Kalkstickstofffabrik bereits 1905 den Betrieb aufgenommen hatte, entstanden vor dem Ersten Weltkrieg auch in Deutschland und in der Schweiz diverse derartige Fabrikanlagen. So etwa in Trostberg, Mühltal, Westeregeln, Knapsack und GrossKayna (Deutschland) sowie Martigny und Gampel (Schweiz). ${ }^{14}$

10 B. Waeser, Die Luftstickstoff-Industrie. Mit besonderer Berücksichtigung der Gewinnung von Ammoniak und Salpetersäure, Leipzig 1922, S. 48-49.

11 Fehr, Stickstofffrage, S. 34-35.

12 N. Caro, Stickstofffrage, S. 46-48; Ders., Die Stickstoffgewinnung im Kriege, in: M. Schwarte (Hg.), Die Technik im Weltkriege, Berlin 1920, S. 540-541; H.H. Franck, Chemie des Kalkstickstoffs, in: H. Grossmann (Hg.), Der Kalkstickstoff in Wissenschaft, Technik und Wirtschaft, Stuttgart 1931, S. 135-140.

13 Fehr, Stickstofffrage, S. 36-37.

14 F. Janke, Geschichte, Technik und Wirtschaft des Kalkstickstoffs, in: H. Grossmann (Hg.), Der Kalkstickstoff in Wissenschaft, Technik und Wirtschaft, Stuttgart 1931, S. 50-52; Abteilung 
Ebenfalls noch vor dem Ersten Weltkrieg konnte das bis heute primär unter der Bezeichnung Haber-Bosch-Verfahren bekannte Hochdruckverfahren im größeren Rahmen verwirklicht werden. Dabei wird Stickstoff unter hohem Druck und in Anwesenheit eines Katalysators mit Wasserstoff zur Reaktion gebracht, wobei Ammoniak entsteht. Das auf den ersten Blick relativ einfach erscheinende Grundprinzip dieses Verfahrens wurde in der Theorie schon früh erkannt. Die Entwicklung und der Betrieb einer Vorrichtung, die eine nennenswerte Ammoniakausbeute hervorbrachte, erwiesen sich allerdings selbst im Labormaßstab als dermaßen schwierig, dass dies erst 1909 dem für die BASF tätigen Chemiker und späteren Nobelpreisträger Fritz Haber (1868-1934) gelang. Er konstruierte eine unter einem Druck von rund 200 Atmosphären arbeitende Zirkulationsapparatur, die einen Osmium-Katalysator enthielt. ${ }^{15}$ Die Übertragung in den industriellen Maßstab erfolgte in den Folgejahren unter der Leitung von Carl Bosch (1874-1940), wobei nicht nur der teure und sehr seltene Osmium- durch einen Eisenkatalysator ersetzt wurde, sondern auch eine Stahlhülle entwickelt werden konnte, die dem im Synthesegas enthaltenen Wasserstoff standhielt. ${ }^{16}$ Beim Kriegsausbruch standen in der BASF bereits erste größere Produktionsanlagen zur Verfügung, in denen das Hochdruckverfahren zur Anwendung kam. Dabei zeigte sich, dass dieses Verfahren im Vergleich zum Lichtbogen- und zum Kalkstickstoffverfahren das mit Abstand energieeffizienteste war. ${ }^{17}$

Die industrielle Fixierung des in der Luft enthaltenen Stickstoffs mittels der drei beschriebenen Verfahren wurde zwar bereits vor dem Ersten Weltkrieg als Weg zur Lösung der globalen Stickstofffrage bzw. zur Überwindung der Abhängigkeit von Chilesalpeter erkannt. Daneben bestand mit der in Gaswerken und Kokereien betriebenen Trockendestillation von Kohle aber auch noch eine vierte Möglichkeit zur Versorgung mit Stickstoffverbindungen. Das beim Erhitzen von

für industrielle Kriegswirtschaft (Hg.), Die Abteilung für industrielle Kriegswirtschaft des eidgenössischen Volkswirtschafts-Departementes 2, Sektion Chemie sowie Handelsabteilung „Chemische Angelegenheiten“, 1915-1919. Übersicht über Tätigkeit und Entwicklung der schweizerischen chemischen Industrie vor und während des Weltkrieges, Bern 1925, S. 46; Lonza AG (Hg.), 50 Jahre Lonza Elektrizitätswerke und chemische Fabriken Aktiengesellschaft, 18971947, o.O. 1947, S. 20.

15 F. Haber, Über die Darstellung des Ammoniaks aus Stickstoff und Wasserstoff. Vortrag, gehalten beim Empfang des Nobelpreises in Stockholm am 2. Juni 1920, in: F. Haber (Hg.), Fünf Vorträge aus den Jahren 1920-1923, Berlin 1924, S. 13-19; D. Stoltzenberg, Fritz Haber. Chemiker, Nobelpreisträger, Deutscher, Jude, Weinheim 1994, S. 151-170; M. Szöllösi-Janze, Fritz Haber, 1868-1934. Eine Biographie, München 1998, S. 175-180.

16 A. von Nagel, Stickstoff. Die technische Chemie stellt die Ernährung sicher, Ludwigshafen 1969, S. 22-35.

17 Fehr, Stickstofffrage, S. 40-41. 
Kohle als Nebenprodukt anfallende Ammoniak, das sich beispielsweise mit Schwefelsäure zum Stickstoffdünger Ammoniumsulfat weiterverarbeiten lässt, war in Deutschland vor dem Ausbruch des Ersten Weltkriegs die neben dem Chilesalpeter am meisten benötigte Stickstoffverbindung überhaupt. ${ }^{18}$ Obschon dieses Verfahren durchaus zur Minderung der Abhängigkeit von Chilesalpeter beitrug, war es zur Lösung der Stickstofffrage ungeeignet. Denn der Ammoniakausstoß war dabei direkt an die Koks- und indirekt an die Stahlproduktion gekoppelt, so dass er nur sehr beschränkt, d.h. nicht entsprechend der Stickstoffnachfrage gesteigert werden konnte. ${ }^{19}$

Die Ressource Stickstoff war nicht nur für die Landwirtschaft, und damit für die Ernährung der Bevölkerung bzw. die Bewahrung von Menschenleben, sondern auch für die Kriegführung, und damit die Zerstörung von Menschenleben, von großer Bedeutung. Praktisch alle militärisch brauchbaren Explosivstoffe, d.h. Sprengstoffe, Schießpulver und Zündmittel, sind selbst Stickstoffverbindungen und werden auch aus solchen hergestellt. Während zur Gewinnung von Schwarzpulver, dem ältesten Schießpulver überhaupt, Salpeter erforderlich ist, wird bei der Produktion der meisten übrigen Explosivstoffe primär Salpetersäure benötigt, mit der dann eine Nitrierung, beispielsweise von Toluol zu Nitrotoluol (TNT) oder von Baumwolle zu Nitrozellulose, vorgenommen werden kann. Die Salpetersäure wurde vor dem Ersten Weltkrieg wiederum hauptsächlich durch Umsetzung von Chilesalpeter mit Schwefelsäure hergestellt. ${ }^{20}$ Explosivstoffe, die auch ohne Stickstoffverbindungen hergestellt werden können, waren mit den Chloratsprengstoffen zwar bereits vor dem Ersten Weltkrieg bekannt. Allerdings entsprachen diese nicht den Anforderungen, die für eine militärische Verwendung zu erfüllen waren und erwiesen sich als zu instabil bzw. zu gefährlich. Sie kamen daher nur in wenigen Nischenbereichen oder zur Streckung anderer Explosivstoffe zu Anwendung. ${ }^{21}$ Da Stickstoffverbindungen für die Kriegführung unverzichtbar, gleichzeitig aber auch für die Landwirtschaft bzw. die Ernährung der Bevölkerung

18 G. Schuchardt, Die technische Gewinnung von Stickstoff, Ammoniak und schwefelsaurem Ammonium, nebst einer Übersicht der deutschen Patente, Stuttgart 1919, S. 219-229.

19 Fehr, Stickstofffrage, S. 76-77, 89.

20 Caro, Stickstoffgewinnung, S. 537-538; A. Schmidt, Die industrielle Chemie in ihrer Bedeutung im Weltbild und Erinnerungen an ihren Aufbau, Berlin 1934, S. 644-650.

21 Ebda., S. 650-652. Gewisse während des Ersten Weltkriegs aufgrund von Rohstoffmangel ausnahmsweise für militärische Zwecke beschaffte Chloratsprengstoffe neigten dazu, sich sogar in vermeintlich sicheren Munitionsdepots ohne jede äußere Einwirkung selbständig zu entzünden. (H. Burkhardt, Dynamit am Gotthard. Sprengstoff in der Schweiz. Isleten, das älteste Industrieareal des Kantons Uri, 1596-2011. Erzverhüttung - Sägereien - Papierfabrik - Sprengstofffabrik. Einblick in die Geschichte der schweizerischen Sprengstoffindustrie, 1873-2011, Baden 2012, S. 88-93.) 
essentiell waren, kann Stickstoff als eine Dual-Use-Ressource von strategischer Bedeutung bezeichnet werden.

\section{Die deutsche Stickstofffrage während des Ersten Weltkriegs}

Die deutsche Volkswirtschaft war unmittelbar vor dem Ersten Weltkrieg besonders stark von der Einfuhr südamerikanischen Salpeters abhängig. Um diese Abhängigkeit zu quantifizieren, bietet es sich an, den deutschen Gesamtverbrauch von gebundenem Stickstoff des Jahres 1913 der tatsächlichen Inlandproduktion der Stickstoffwirtschaft gegenüberzustellen. Dabei zeigt sich, dass der Bedarf von rund 225.000 Jahrestonnen Stickstoff von der inländischen Industrie selbst im bestmöglichen Fall - d.h. unter der Annahme, dass keine Exporte stattfinden und die Produktion nicht gedrosselt wird - zu höchstens 57 Prozent hätte gedeckt werden können. Die Auslandabhängigkeit betrug also mindestens 43 Prozent. Der mit Abstand größte Teil der inländischen Produktion stammte aus Kokereien und Gaswerken. Durch die Luftstickstofffixierung hätten 1913 selbst im bestmöglichen Fall höchstens acht Prozent des deutschen Bedarfs an Stickstoffverbindungen gedeckt werden können. Bei diesen Berechnungen ist noch zu berücksichtigen, dass sie sich lediglich auf die Friedenszeit beziehen und daher von einem schon fast verschwindend geringen Streitkräftebedarf ausgehen, der im Kriegsfall stark zunehmen würde. Das tatsächliche Ausmaß der Abhängigkeit Deutschlands von Chilesalpetereinfuhren dürfte daher noch wesentlich größer gewesen sein. ${ }^{22}$ Dennoch waren im Vorfeld des Ersten Weltkriegs praktisch keine wirtschaftlichen Vorsorgemaßnahmen getroffen worden. ${ }^{23}$

Unmittelbar nach dem Ausbruch des Ersten Weltkriegs war klar, dass dieser nicht nur an der militärischen Front, sondern auch als „offensiver Wirtschaftskrieg“ geführt wurde, bei dem „beide Parteien bestrebt [waren] den Gegner vom [...] Welthandel abzuschneiden.“24 $\mathrm{Zu}$ den wirtschaftlichen Kampfmaßnahmen der Entente zählte insbesondere die Verwirklichung der von der britischen Marine schon lange vor dem Kriegsausbruch vorbereiteten Seeblockade. ${ }^{25}$ Diese schnitt

22 Fehr, Stickstofffrage, S. 57-59, 177.

23 L. Burchardt, Friedenswirtschaft und Kriegsvorsorge. Deutschlands wirtschaftliche Rüstungsbestrebungen vor 1914, Boppard am Rhein 1968, S. 245-246.

24 Hardach, Weltkrieg, S. 19.

25 D.M. Segesser, Der Erste Weltkrieg in globaler Perspektive, Wiesbaden 2010, S. 143; A. Offer, The First World War. An Agrarian Interpretation, Oxford 1989, S. 285-317. 
die Mittelmächte nach kurzer Zeit weitgehend vom Weltmarkt ab. ${ }^{26}$ Abgesehen von geringen Mengen militärisch erbeuteter Chemikalien und Einfuhren von in neutralen Nachbarstaaten produzierten Stoffen gelangten dadurch praktisch keine Stickstoffverbindungen mehr nach Deutschland. ${ }^{27}$ Die Einfuhren von Chilesalpeter fielen sogar vollständig aus. ${ }^{28}$ Damit wurde die im 19. Jahrhundert von Wissenschaftlern wie Crookes prognostizierte langsame globale Verknappung der für die Ernährung der Bevölkerung so wichtigen Ressource Stickstoff in Deutschland beim Beginn des Ersten Weltkriegs auf einen Schlag Realität.

Bereits vor dem Kriegsbeginn war klar, dass der Heeresbedarf, der in Friedenszeiten praktisch gar nicht ins Gewicht fiel, während eines Kriegs stark zunehmen würde. Das nach dem Kriegsbeginn feststellbare tatsächliche Ausmaß der Bedarfssteigerung übertraf dann aber doch alle Erwartungen. Mit dem Übergang vom Bewegungs- zum Stellungskrieg zeigte sich, dass der Munitionsbedarf bereits Ende 1914 vier- bis sechsmal höher als ursprünglich erwartet war. ${ }^{29}$ An gewissen Tagen soll sogar mehr Munition verbraucht worden sein ,als im ganzen Krieg 1870/71“. ${ }^{30}$ Daraus resultierte sowohl bei den Mittelmächten als auch seitens der Entente eine Munitionskrise. ${ }^{31}$ Obschon beide Kriegsparteien davon betroffen waren, unterschied sich die Situation der Entente dadurch von derjenigen der Mittelmächte, dass letztere aufgrund der Blockade nicht über die für eine starke Steigerung der Munitionserzeugung erforderlichen Rohstoffe verfügten.

Zusammenfassend lässt sich festhalten, dass die deutsche Kriegswirtschaft beim Kriegsausbruch vor dem geradezu existentiellen Problem stand, dass der Bedarf nach der Ressource Stickstoff - die sowohl für die Kriegführung als auch für die Ernährung der Bevölkerung unentbehrlich war - außerordentlich stark zunahm, während das Angebot gleichzeitig um rund die Hälfte einbrach. Wollte Deutschland eine Hungersnot vermeiden und den Krieg nicht frühzeitig aus

26 Michalka, Kriegsrohstoffbewirtschaftung, S. 486.

27 Fehr, Stickstofffrage, S. 68-69; W. Eucken, Die Stickstoffversorgung der Welt. Eine volkswirtschaftliche Untersuchung, Stuttgart 1921, S. 88-89; Deutsches Bundesarchiv-Militärarchiv (BArch-MA), MSg 2760 a, Denkschrift der Zentralstelle für Fragen der Chemie des Kriegsministeriums zum neuen Munitionsprogramm vom 3. Februar 1916, 5. Februar 1916, Auszug 2.

28 Deutsches Bundesarchiv (BArch), R 432466 a, Schreiben des Ministeriums für Landwirtschaft, Domänen und Forsten an den Reichskanzler (Großes Hauptquartier) vom 02.11.1914, S. 1.

29 Fehr, Stickstofffrage, S. 80-81; W. von Moellendorff, Das Tagebuch Wichard v. Moellendorffs vom 3. August bis zum 14. Oktober 1914, in: Tradition. Zeitschrift für Firmengeschichte und Unternehmerbiographie 2, 1971, S. 90.

30 O. Goebel, Deutsche Rohstoffwirtschaft im Weltkrieg einschließlich des Hindenburg-Programms, Stuttgart 1930, S. 19.

31 B. Thoss, Munitionskrise, in: G. Hirschfeld/G. Krumeich/I. Renz (Hg.), Enzyklopädie Erster Weltkrieg, Paderborn 2004, S. 727-728. 
Munitionsmangel verlieren, musste es die nun auf den nationalen Rahmen begrenzte Stickstofffrage so rasch als möglich lösen. Es würde den Rahmen des vorliegenden Artikels sprengen, die kriegswirtschaftlichen Maßnahmen Deutschlands zur Lösung der Stickstofffrage in extenso auszuführen, zumal dies an anderer Stelle bereits erfolgt ist. ${ }^{32}$ Im Folgenden wird daher lediglich zusammenfassend dargestellt, wie und mit welchem Erfolg die deutsche Kriegswirtschaft das Versorgungsproblem während des Ersten Weltkriegs zu lösen versuchte.

In Anbetracht der Tatsache, dass in Deutschland vor dem Krieg kaum wirtschaftliche Vorsorgemaßnahmen getroffen worden waren, erstaunt es nicht, dass die Initiative für kriegswirtschaftliche Maßnahmen auch nach dem Kriegsbeginn nicht vom Staat, sondern von der Privatwirtschaft ausging. Nachdem der Industrielle Walther Rathenau (1867-1922) den preußischen Kriegsminister Erich von Falkenhayn (1861-1922) im August 1914 auf den dringenden Handlungsbedarf im Bereich der Rohstoffversorgung aufmerksam gemacht hatte, wurde er von diesem sogleich mit dem Aufbau und der Leitung der Kriegsrohstoffabteilung (KRA) im preußischen Kriegsministerium beauftragt. Diese Behörde, die fortan für die gesamte Rohstoffbewirtschaftung in Deutschland zuständig war, nahm sich bereits in den ersten Tagen ihres Bestehens auch der „Salpeterversorgung “ an. ${ }^{33}$

Nachdem sich die KRA im Gründungsmonat August 1914 zunächst einen Überblick verschaffen musste und eher kurzfristig wirksame Maßnahmen, wie etwa Beschlagnahmungen, beschlossen hatte, gründete sie im September 1914 die Kriegschemikalien AG und leitete eine Ausdehnung der inländischen Produktion der für die Explosivstofferzeugung unentbehrlichen Salpetersäure in die Wege, wobei sie auf das Verfahren der Ammoniakoxidation setzte. ${ }^{34}$ Dabei wird Ammoniak in Anwesenheit eines Katalysators mit dem in der Luft enthaltenen Sauerstoff zur Reaktion gebracht, wobei Stickoxide entstehen, die mit Wasser wiederum zu Salpetersäure umgesetzt werden können. Dieses Verfahren war von den chemischen Werken der Steinkohlenzeche Lothringen in Gerthe bei Bochum

32 Siehe dazu: Fehr, Stickstofffrage, S. 64-121; Szöllösi-Janze, Haber, S. 273-316; Dies., Berater, Agent, Interessent? Fritz Haber, die BASF und die staatliche Stickstoffpolitik im Ersten Weltkrieg, in: Berichte zur Wissenschaftsgeschichte 2-3, 1996, S. 105-117; Dies., Losing the War but Gaining Ground: The German Chemical Industry During World War I, in: J. Lesch (Hg.), The German Chemical Industry in the Twentieth Century, Dordrecht 2000, S. 91-121; J.A. Johnson, Die Macht der Synthese (1900-1925), in: W. Abelshauser (Hg.), Die BASF. Eine Unternehmensgeschichte, München 2002, S. 168-181.

33 Michalka, Kriegsrohstoffbewirtschaftung, S. 487; W. Brenner, Walther Rathenau. Deutscher und Jude, München 2005, S. 313-315; C. Schölzel, Walther Rathenau. Eine Biographie, Paderborn 2006, S. 175-177; Moellendorff, Tagebuch, S. 80-82.

34 Moellendorff, Tagebuch, S. 87-91. 
bereits lange vor dem Kriegsausbruch im industriellen Rahmen betrieben worden, trug damals aber nur unwesentlich zur deutschen Gesamtversorgung bei. Außerdem wurde in der Anlage in Gerthe äußerst teures und seltenes Platin als Katalysator verwendet. Im Frühjahr 1914 hatte die BASF einen wesentlich günstigeren und einfacher $\mathrm{zu}$ beschaffenden Eisenoxid-Wismutoxid-Manganoxid-Katalysator entwickelt, der nun in den neu zu errichtenden Produktionsanlagen eingesetzt werden sollte. ${ }^{35}$ Noch im September 1914 beauftragte das Preußische Kriegsministerium die BASF zur schnellstmöglichen Errichtung umfangreicher Fabrikanlagen und sicherte ihr im November 1914 vertraglich Staatszuschüsse und diverse weitere Unterstützungsmaßnahmen zu. Neben der BASF errichteten bald auch Bayer, Agfa, die österreichische Kriegsverwaltung und die Holzverkohlungs-AG in Konstanz Ammoniakoxidationsanlagen nach dem BASF-Verfahren, wobei zumindest im Fall der deutschen Firmen ebenfalls Verträge mit dem Kriegsministerium abgeschlossen wurden. Der Bau der Oxidationsanlagen wurde in der Folge mit Hochdruck vorangetrieben, so dass die ersten bereits im Februar 1915 den Betrieb aufnehmen konnten. ${ }^{36}$ Im Dezember 1915 erreichte die Salpetersäureerzeugung in den neuen Oxidationsanlagen das im Herbst 1914 festgelegte Soll von 4.300 Tonnen Stickstoff pro Monat und wurde anschließend weiter gesteigert. Wie eine Analyse der Lagerbestände von Salpeter im Inland zeigt, setzte die Salpetersäureproduktion gerade noch rechtzeitig ein, damit der Krieg fortgesetzt werden konnte. Wenige Monate später wären die letzten Reserven von Chilesalpeter aufgebraucht gewesen..$^{37}$

Mit dem Bau der Ammoniakoxidationsanlagen konnte zumindest der Grundbedarf der Explosivstoffindustrie nach Salpetersäure bis auf weiteres gedeckt werden. Die eigentliche Stickstofffrage, d.h. die Sicherstellung der Versorgung des Landes mit gebundenem Stickstoff, konnte damit aber in keiner Weise gelöst werden. Dies kam daher, dass die Ammoniakoxidation lediglich eine Stickstoffverbindung in eine andere umwandelt, die Menge an gebundenem Stickstoff insgesamt aber nicht erhöht. Da die Salpetersäure in erster Linie der Kriegführung zugute kam, kann sogar gesagt werden, dass durch den Ausbau der Oxidationsanlagen die rare Ressource Stickstoff vom zivilen in den militärischen Bereich umverteilt wurde. Sollte auch die Landwirtschaft ausreichend versorgt werden, musste die Produktion primärer Stickstoffverbindungen ebenfalls dringend vor-

35 Nagel, Stickstoff, S. 56-59.

36 Moellendorff, Tagebuch, S. 89-91; Szöllösi-Janze, Berater, S. 107-108; A. Mittasch, Salpetersäure aus Ammoniak. Geschichtliche Entwicklung der Ammoniakoxydation bis 1920, Weinheim 1953, S. 118-123.

37 Fehr, Stickstofffrage, S. 86-87. 
angetrieben werden. ${ }^{38}$ Es verwundert daher nicht, dass in diesem Bereich ausgerechnet das Preußische Landwirtschaftsministerium die Initiative ergriff. Dieses setze dabei sowohl auf das Hochdruckverfahren der BASF, als auch auf das Kalkstickstoffverfahren, wobei letzteres zunächst priorisiert wurde. ${ }^{39}$ Das Ministerium einigte sich im Dezember 1914 mit der AG für Stickstoffdünger in Knapsack bei Köln, an der die Farbwerke Hoechst eine Beteiligung hielten, ${ }^{40}$ und mit der BASF über die mit staatlichen Darlehen unterstütze Erweiterung ihrer Produktion von Kalkstickstoff bzw. Ammoniak. ${ }^{41}$ Im Frühling 1915 schloss zudem das Reichsschatzamt mit den Bayerischen Stickstoffwerken - trotz des erbitterten Widerstands der BASF - einen Vertrag über die Errichtung und den Betrieb von staatlichen Kalkstickstofffabriken, sogenannten „Reichsstickstoffwerken“, sowie über den Ausbau bereits bestehender Kalkstickstoffwerke ab. ${ }^{42}$ Im selben Zeitraum verpflichtete sich außerdem die Lonza-Werke elektrochemische Fabriken $\mathrm{GmbH}$ in Waldshut, die zuvor lediglich Calciumcarbid hergestellt hatte, vertraglich zur Erzeugung von Kalkstickstoff. ${ }^{43}$ Durch diese Maßnahmen sollte die Kalkstickstoffproduktion in Deutschland insgesamt von rund 10.000 auf 86.000 Tonnen Stickstoff pro Jahr und die Herstellung nach dem Hochdruckverfahren von 7.000 auf 56.000 Jahrestonnen Stickstoff gesteigert werden. ${ }^{44}$

Neben dem Kalkstickstoff- und dem Hochdruckverfahren wurde in Rhina bei Laufenburg in Baden und in Zschornewitz dank staatlicher Unterstützung auch das energieintensive Lichtbogenverfahren verwirklicht. Mit einer angestrebten Produktion von lediglich 400 Jahrestonnen Stickstoff, die während des gesamten Kriegs nie erreicht wurden, trugen sie allerdings nur marginal zur deutschen Stickstoffversorgung bei. ${ }^{45}$ Der Ammoniakausstoß durch Trockendestillation von Kohle war im Vergleich dazu zwar wesentlich höher. Insgesamt nahm die Produktion der Kokereien und Gaswerke aber während der gesamten Kriegsdauer

38 Ebda., S. 88.

39 BArch, R 432466 a, Protokoll der Sitzung im Ministerium für Landwirtschaft, Domänen und Forsten vom 20.10.1914, S. 11.

40 Zur AG für Stickstoffdünger S. Knetsch, Das konzerneigene Bankinstitut der Metallgesellschaft im Zeitraum von 1906 bis 1926, Stuttgart 1998, S. 158.

41 BArch, R 2 43039, Protokoll der Sitzung des Königlichen Staatsministeriums vom 31.12.1914, S. 2.

42 K. Helfferich, Der Weltkrieg 2, Vom Kriegsausbruch bis zum uneingeschränkten U-Bootkrieg, Berlin 1919, S. 121; R. Roth, Staat und Wirtschaft im Ersten Weltkrieg. Kriegsgesellschaften als kriegswirtschaftliche Steuerungsinstrumente, Berlin 1997, S. 212.

43 Eucken, Stickstoffversorgung, S. 103.

44 Fehr, Stickstofffrage, S. 151-152.

45 BArch, R 8729 287, Bericht von Leutnant Holm der KCA über Besuche bei den Werken Zschornewitz und Rhina vom 23.11.1916, S. 1. 
kontinuierlich ab, wodurch sie die Versorgungslage während des Kriegs im Vergleich zur Vorkriegszeit nicht verbesserten, sondern im Gegenteil sogar verschlechterten. ${ }^{46}$

Die anfängliche Priorisierung des Kalkstickstoffverfahrens gegenüber dem Hochdruckverfahren endete spätestens, als das Kriegsministerium im Frühjahr 1916 erneut einen weiteren Ausbau der Produktion primärer Stickstoffverbindungen veranlasste und dabei ausschließlich auf die BASF und deren Hochdruckverfahren setzte. Diese Produktionssteigerungen sollten mit dem Bau eines neuen Werks in Leuna bei Merseburg mit einer Kapazität von 30.000 Tonnen Stickstoff pro Jahr verwirklicht werden. ${ }^{47} \mathrm{Im}$ Zusammenhang mit dem Hindenburgprogramm, das eine Verdoppelung der Schießpulverproduktion vorsah, wurde das Produktionsziel zunächst auf 130.000 Jahrestonnen Stickstoff vervierfacht, um es 1918 schließlich auf 200.000 Jahrestonnen Stickstoff zu erhöhen. ${ }^{48}$ Die Gründe für die Abkehr von der Förderung des Kalkstickstoff- zugunsten des Hochdruckverfahrens lagen zum einen darin, dass der Munitionsverbrauch der Truppe im Verlauf des Kriegs immer stärker zunahm und zur Explosivstofferzeugung in erster Linie Salpetersäure benötigt wurde, die wiederum direkter und billiger aus Ammoniak als aus Calciumcyanamid hergestellt werden konnte. Zum anderen führte die durch das Hindenburgprogramm mit verursachte landesweite Energie-, Transport- und Arbeitskräftekrise dazu, dass der höhere Energie- und Arbeitskräftebedarf des Kalkstickstoffverfahrens bei den Entscheidungsträgern besonders ins Gewicht fiel. ${ }^{49}$ Der Strategiewechsel ist auch in den effektiven Produktionszahlen klar ersichtlich. Konnten die Kalkstickstofffabriken ihren Ausstoß von rund 15.000 Tonnen Stickstoff im Jahr 1915 auf über 34.000 Tonnen im Jahr 1916 noch mehr als verdoppeln, verharrte er in den folgenden Jahren auf einem Wert um 35.000 Jahrestonnen; anders die BASF mit dem Hochdruckverfahren, die 1915 mit rund 12.000 Tonnen Stickstoff noch deutlich weniger als die Kalkstickstoffindustrie erzeugte, ihre Produktion in den Folgejahren aber auf

46 Fehr, Stickstofffrage, S. 89-90, 179.

47 L.F. Haber, The Chemical Industry, 1900-1930. International Growth and Technological Change, Oxford 1971, S. 202-203.

48 BASF Unternehmensarchiv, A 18 23, Vertrag zwischen dem Reichskanzler (Reichsschatzamt) und der Badischen Anilin- \& Soda-Fabrik zu Ludwigshafen, 1./19. Dezember 1916, S. 1-2; W. Voigtländer-Tetzner, Die Bindung des Luftstickstoffs, 1895-1940 1, 1895-1918. Unveröffentlichtes Manuskript im BASF Unternehmensarchiv, Ludwigshafen 1940, S. 303.

$49 \mathrm{Fehr}$, Stickstofffrage, S. 113-115; E. Ludendorff, Urkunden der Obersten Heeresleitung über ihre Tätigkeit 1916/19, Berlin 1920, S. 208. 
51.000 Tonnen (1916), 75.000 Tonnen (1917) und schließlich sogar 94.000 Tonnen (1918) erhöhte. ${ }^{50}$

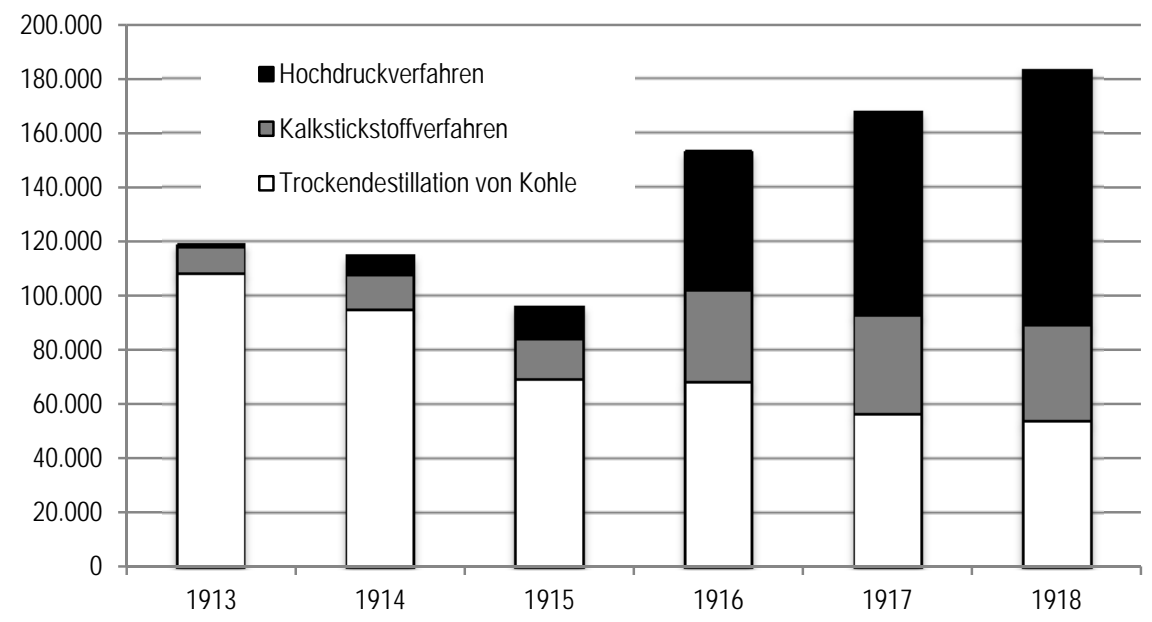

Abb.1: Die Gesamtproduktion der deutschen Stickstoffwirtschaft 1913-1918 in Tonnen Stickstoff ${ }^{51}$

Die Gesamterzeugung der deutschen Stickstoffwirtschaft sank in den ersten beiden Kriegsjahren von ursprünglich rund 119.000 Tonnen vor dem Krieg auf 115.000 Tonnen (1914) und 96.000 Tonnen (1915), um im Jahr 1916 nicht nur wieder anzusteigen, sondern mit 153.000 Tonnen Stickstoff auch die Vorkriegsproduktion zum ersten Mal überhaupt zu übertreffen und in den letzten beiden Jahren 169.000 Tonnen (1917) und schließlich 184.000 Tonnen Stickstoff (1918) zu erreichen (siehe Abb. 1). Wird berücksichtigt, dass der Bedarf nach Stickstoffverbindungen bereits vor dem Krieg, d.h. ohne den gesteigerten militärischen Bedarf, über 200.000 Jahrestonnen Stickstoff betragen hat und der Bedarf im Verlauf des Kriegs auf weit über 300.000 Jahrestonnen angestiegen sein dürfte, wird deutlich, dass die deutsche Kriegswirtschaft zu keinem Zeitpunkt des Ersten Weltkriegs dazu imstande war, den Bedarf nach Stickstoffverbindungen auch nur annähernd zu decken. Insgesamt bestand ein jährlicher Fehlbetrag von ungefähr 100.000 Tonnen Stickstoff. ${ }^{52}$ Eingespart wurde dieser in erster Linie in der Landwirtschaft, die in gewissen Düngejahren nur noch rund ein Drittel ihres Vor-

50 Fehr, Stickstofffrage, S. 116, 180.

51 Ebda., S. 180.

52 Ebda., S. 116-118, 180. 
kriegsbedarfs an Stickstoffdünger zugeteilt erhielt. ${ }^{53}$ Hinsichtlich der Zeit nach dem Ersten Weltkrieg, in welcher der Streitkräftebedarf weitgehend wegfallen musste, ist aber festzuhalten, dass Deutschland bei Kriegsende über eine Produktionsinfrastruktur verfügte, mit welcher der zivile Vorkriegsbedarf der Landwirtschaft von rund 200.000 Tonnen Stickstoff pro Jahr größtenteils gedeckt werden konnte. Es kann somit argumentiert werden, dass mit den kriegswirtschaftlichen Anstrengungen während des Ersten Weltkriegs zumindest die ursprüngliche, im 19. Jahrhundert von Wissenschaftlern wie Crookes aufgeworfene Stickstofffrage, in Deutschland - begrenzt auf den nationalen Rahmen - durchaus gelöst wurde. Damit war bewiesen, dass dichtbesiedelte, industrialisierte Staaten mit einer entsprechenden Produktionsinfrastruktur und genügend Energie auch unabhängig von Chilesalpetereinfuhren bestehen konnten. ${ }^{54}$ Die Stickstofffrage war zu einer Energiefrage geworden.

\section{Die Stickstoffversorgung der Schweiz während des Ersten Weltkriegs}

Der neutrale Kleinstaat Schweiz war während des Ersten Weltkriegs nicht direkt in militärische Kampfhandlungen verstrickt. ${ }^{55}$ Da der Krieg aber nicht nur mit militärischen Mitteln, sondern auch als Wirtschaftskrieg geführt wurde, wäre es falsch zu behaupten, die Schweiz sei nicht in den Krieg involviert gewesen. ${ }^{56}$ Aufgrund ihrer ausgeprägten ökonomischen Verflechtung war auch sie stark von der weitgehenden Zerstörung des internationalen Handelssystems betroffen, was sich nicht nur darin äußerte, dass im Export gewisse Absatzmärkte wegfielen, sondern primär auch darin, dass die Importe von Rohstoffen und Lebensmitteln einbrachen. ${ }^{57}$ So sanken die Lebensmitteleinfuhren in die Schweiz beispielsweise von

53 F. Aereboe, Der Einfluss des Krieges auf die landwirtschaftliche Produktion in Deutschland, Stuttgart 1927, S. 43.

54 Fehr, Stickstofffrage, S. 158-159.

55 J. Tanner/V. Groebner/S. Guex, Einleitung: Kriegswirtschaft und Wirtschaftskriege. Forschungsperspektiven und Untersuchungsfelder, in: V. Groebner/S. Guex/J. Tanner (Hg.), Kriegswirtschaft und Wirtschaftskriege, Zürich 2008, S. 16.

56 S. Fehr, Die Vulnerabilität der chemieindustriellen Basis von Rüstung und Krieg in der Schweiz während des Ersten Weltkriegs, in: R. Jaun/D. Rieder (Hg.), Schweizer Rüstung. Politik, Beschaffungen und Industrie im 20. Jahrhundert, Baden 2013, S. 33.

57 R. Rossfeld/T. Straumann, Zwischen den Fronten oder an allen Fronten? Eine Einführung, in: Dies. (Hg.), Der vergessene Wirtschaftskrieg. Schweizer Unternehmen im Ersten Weltkrieg, Zürich 2008, S. 20-25. 
1.736 Tonnen im Jahr 1913 auf 553 Tonnen im Jahr 1918. Das entsprach einem Rückgang um 68 Prozent. Kaum weniger stark war der Einbruch bei den Importen von Rohstoffen, die von 5.692 Tonnen im Jahr 1913 auf 2.780 Tonnen im Jahr 1918 (oder um 49 Prozent) abnahmen..$^{58}$ Die Schweizer Wirtschaft war vom Wirtschaftskrieg aber nicht nur passiv betroffen, sondern belieferte die kriegführenden Mächte auch aktiv im großen Rahmen mit kriegswichtigen Erzeugnissen wie zum Beispiel Munitionsbestandteilen (Stahlmäntel für Artilleriegeschosse, Geschossteile aus Kupfer, Zink und Aluminium sowie Schrapnellzünder) oder Materialien „für den Bau von Bunkern, Schützengräben und Unterständen“ an der Front. ${ }^{59}$

Was ihre eigene Versorgung betrifft, importierte die Schweiz vor dem Krieg, ähnlich wie Deutschland, große Mengen von Stickstoffverbindungen. Die schweizerische Landwirtschaft verwendete in der Vorkriegszeit neben Ammoniumsulfat, das auf der Basis der Trockendestillation von Kohle im Inland produziert wurde, vor allem Chilesalpeter. ${ }^{60}$ Insgesamt dürfte der landwirtschaftliche Verbrauch unmittelbar vor Kriegsbeginn jährlich über 1.000 Tonnen Salpeter betragen haben, was ca. 160 Jahrestonnen Stickstoff entsprach. ${ }^{61}$ Zudem waren auch in der Schweiz praktisch alle militärischen Explosivstoffe Stickstoffverbindungen, die zumeist durch Nitrierung mit Salpetersäure hergestellt wurden. ${ }^{62}$ Bis 1911 führte die Schweiz rund 30 Prozent ihres Gesamtverbrauchs von Salpetersäure aus dem Ausland ein und produzierte die übrigen 70 Prozent in der Chemischen Fabrik Uetikon am Zürichsee, indem sie Chilesalpeter mit Schwefelsäure versetzte. Da in den Jahren 1912 und 1913 in der Schweiz erste nach dem Lichtbogenverfahren arbeitende Salpetersäurefabriken die Produktion aufnahmen, verringerte sich die Importabhängigkeit zwar leicht, insgesamt basierte bei Beginn des Ersten Weltkriegs aber dennoch der überwiegende Teil der Salpetersäureversorgung auf der Zufuhr von Chilesalpeter. ${ }^{63}$

Da die Schweiz ein Binnenland ist, konnte sie den Chilesalpeter nicht direkt einschiffen, sondern musste ihn indirekt über Drittstaaten beziehen. Vor dem Ausbruch des Ersten Weltkriegs war Deutschland der wichtigste Zwischenhändler. Kleinere Mengen wurden aber auch über Frankreich, Belgien und die Nieder-

58 T. Geering, Handel und Industrie der Schweiz unter dem Einfluss des Weltkriegs, Basel 1928, S. 22-23.

59 Ebda., S. 574-578; Rossfeld/Straumann, Fronten, S. 26. Siehe auch den Beitrag von Roman Rossfeld in diesem Band.

60 Schweizerisches Bundesarchiv (BAR), E 7350 1000/1104, Bd. 42 (q), Sektion Chemie, Die Versorgung des Landes mit Stickstoffprodukten, 7. August 1918, S. 1-2.

61 Abteilung für industrielle Kriegswirtschaft, Abteilung, S. 42; Fehr, Stickstofffrage, S. 176.

62 Fehr, Vulnerabilität, S. 36.

63 Abteilung für industrielle Kriegswirtschaft, Abteilung, S. 40-41. 
lande bezogen. Insgesamt führte die Schweiz 1913 rund 2.699 Tonnen Chilesalpeter ein, wovon rund 40 Prozent an die Industrie und 60 Prozent an die Landwirtschaft gingen. Im ersten Kriegsjahr 1914 brachen diese Importe auf 1.828 Tonnen und 1915 sogar bis auf bescheidene 140 Tonnen ein, um anschließend auf einem niedrigen Niveau von 343 Tonnen (1916), 259 Tonnen (1917) und 541 Tonnen (1918) zu verharren. ${ }^{64}$ Die Sektion Chemie der Abteilung für industrielle Kriegswirtschaft hielt dazu fest: „Bei Ausbruch des Krieges wurden die Importe an primären Stickstoffverbindungen immer kleiner, um schliesslich beinahe auf Null zu sinken. Die Schweiz musste sich also in ihrem Stickstoffbedarfe selber genügen. Die erste Folge des Ausfalls von Importen an Stickstoffverbindungen war eine unzulängliche künstliche Düngung seitens der Landwirtschaft.“ Und weiter: „In Summa ergibt sich das Bild, dass die Abhängigkeit der Schweiz vom Auslande bezüglich primärer Stickstoffverbindungen sich während des Krieges gerächt hat.“" ${ }^{65}$ Der Einbruch der Importe dürfte allerdings weniger auf eine bewusste Isolation der Schweiz als auf den Umstand zurückzuführen sein, dass Deutschland als bislang wichtigster Zwischenhändler aufgrund der durch die Entente verhängten Blockade ausfiel. Zudem dürften auch die übrigen Staaten die für die Kriegführung so bedeutsame Ressource in erster Linie für den eigenen Gebrauch zurückbehalten haben. ${ }^{66}$

Die Situation der Schweiz lässt sich somit zumindest teilweise mit derjenigen Deutschlands vergleichen, wenn auch mit dem gewichtigen Unterschied, dass die Schweizer Armee nicht in Kampfhandlungen verstrickt war, schon gar keine Materialschlachten zu bestehen hatte und somit auch nicht einen auch nur annähernd so hohen Munitionsverbrauch wie die deutschen Streitkräfte aufwies. Unterschiede bestanden auch bei der Lösung der Stickstofffrage. Während die deutsche Kriegswirtschaft zur Gewinnung primärer Stickstoffverbindungen auf das Kalkstickstoff- und das Hochdruckverfahren setzte, die Salpetersäure mittels Ammoniakoxidation produzierte und das Lichtbogenverfahren praktisch gar nicht berücksichtigte, kam in der Schweiz für die Gewinnung von Salpetersäure ausschließlich das Lichtbogenverfahren und für die Produktion von Stickstoffdünger - nebst der Trockendestillation von Kohle - ausschließlich das Kalkstickstoffverfahren zur Anwendung. Anlagen zur Ammoniakoxidation oder für das Hochdruckverfahren gab es in der Schweiz während des Kriegs keine. ${ }^{67}$

64 Ebda., S. 41-42, 51.

65 BAR, E 7350 1000/1104, Bd. 42 (q), Sektion Chemie, Die Versorgung des Landes mit Stickstoffprodukten, 7. August 1918, S. 4-5.

66 Fehr, Vulnerabilität, S. 37.

67 Ebda., S. 37-38. 
Mit der Société des Produits Azotés in Martigny und dem Werk Gampel der Lonza AG, die beide die Wasserkraft der Alpen als Energiequelle nutzten, verfügte die Schweiz bei Kriegsbeginn bereits über eine funktionierende Kalkstickstoffindustrie, die mit einer Produktion von 8.450 Tonnen im Jahr 1913 die ausgefallenen Chilesalpetereinfuhren gleich mehrfach hätte kompensieren können. Während des Kriegs stiegen die Produktionszahlen mit 13.237 Tonnen (1915), 19.863 Tonnen (1916), 16.977 Tonnen (1917) und 18.208 Tonnen Calciumcyanamid im Jahr 1918 stark an. Theoretisch sollte in der Schweizer Landwirtschaft somit zu keinem Zeitpunkt eine Stickstoffknappheit bestanden haben. Tatsächlich ging die Kalkstickstoffproduktion jedoch während der gesamten Kriegsdauer mehrheitlich in den Export, weil die Schweizer Bauern dem ihnen unbekannten Dünger zunächst mit Misstrauen begegneten. ${ }^{68}$ Da der wesentlich beliebtere Chilesalpeter jedoch nicht mehr verfügbar war und sich auch kein baldiges Ende des Kriegs abzeichnete, hatte die Landwirtschaft letztlich keine andere Wahl, als sich an den neuen Dünger zu gewöhnen. So kam es, „dass der vor dem Kriege vom schweizerischen Bauer verschmähte Kalkstickstoff eine immer wachsende Verwendung fand“. ${ }^{69}$ Somit kann festgestellt werden, dass die Stickstoffversorgung der Schweizer Landwirtschaft während des Ersten Weltkriegs nach einer kurzen Angewöhnungsphase weitgehend sichergestellt war.

Da in der Schweiz im Gegensatz zu Deutschland keine Anlagen zur Umwandlung von Kalkstickstoff zu Salpetersäure existierten, konnte die Explosivstoffindustrie bzw. die Schweizer Armee diese geradezu im Überfluss verfügbare Stickstoffverbindung nicht nutzen. Stattdessen war sie nach dem Ausfall der Salpetereinfuhren auf die bereits erwähnten, nach dem Lichtbogenverfahren produzierenden Salpetersäurefabriken angewiesen. Die erste dieser Fabriken, das Werk Chippis der Aluminium-Industrie AG Neuhausen (AIAG), war ursprünglich eine reine Aluminiumfabrik, welche die fabrikeigenen Wasserkraftwerke ab 1910 im Sinne einer Diversifikation zusätzlich für die Produktion von Salpetersäure nach dem Lichtbogenverfahren von Ignacy Moscicki (1867-1946) nutzen wollte. Dazu baute sie zunächst einen Versuchsbetrieb und ab 1911 eine definitive Fabrik, die ab 1913 in vollem Betrieb stand..$^{70}$ Das Salpetersäurewerk der Nitrum AG befand sich in Bodio und nutzte für seine energieintensive Produktion, genauso

68 Abteilung für industrielle Kriegswirtschaft, Abteilung, S. 46, 58-59.

69 BAR, E 7350 1000/1104, Bd. 42 (q), Sektion Chemie, Die Versorgung des Landes mit Stickstoffprodukten, 07.08.1918, S. 4.

70 Aluminium-Industrie-AG, Geschichte der Aluminium-Industrie-Aktien-Gesellschaft Neuhausen, 1888-1938 1, Die Jahre von 1888 bis 1920, Neuhausen 1942, S. 130-131. Zu Ignacy Moscicki siehe R. Piosik, Die Entwicklung der großtechnischen Luftverbrennung durch Prof. Ignacy Moscicki - polnischer Chemiker und Staatspräsident, in: CHEMKON 4, 1995, S. 159-162. 
wie die bereits behandelten Fabriken in Chippis, Gampel und Martigny, die in den Alpen reichlich verfügbare, günstige Wasserkraft. ${ }^{71}$ Die Fabrik war ursprünglich ein Ableger der deutschen Elektrochemischen Werke Bitterfeld $\mathrm{GmbH}, \mathrm{zu}$ der auch das ebenfalls nach dem Lichtbogenverfahren produzierende Werk im deutschen Rhina gehörte. ${ }^{72} 1915$ wurde die Nitrum AG in einen deutschen Teil mit dem Werk Rhina, im Besitz der AEG, der Elektrochemischen Werke Bitterfeld und der Deutschen Bank sowie in einen schweizerischen Teil mit dem Werk Bodio, im Besitz der Schweizerischen Kreditanstalt, aufgespalten. ${ }^{73}$

Zusammen erzielten die schweizerischen Salpetersäurewerke 1913, in reiner Salpetersäure gerechnet, eine Produktion von 1.260 Tonnen, die im Verlauf des Kriegs auf 1.444 Tonnen (1914 und 1915), 1.491 Tonnen (1916), 2.093 Tonnen (1917) und 2.515 Tonnen (1918) zunahm. Damit konnte in den Jahren 1913-1915 nicht nur der Inlandbedarf gedeckt, sondern sogar Salpetersäure exportiert werden. ${ }^{74}$ In den Jahren 1916 bis 1918 beschlagnahmte das Eidgenössische Militärdepartement allerdings „die gesamte schweizerische Salpetersäureproduktion für die Sprengstoff-Produktion“ “. ${ }^{75}$ In den Folgejahren konnten daher weder nennenswerte Mengen von Salpetersäure aus der Schweiz ausgeführt werden, noch war die zivile Industrie in der Schweiz ausreichend damit versorgt. ${ }^{76}$ Immerhin gelang es dem zivilen Sektor in den Jahren 1916/17 geringe Mengen Salpetersäure aus Frankreich einzuführen, was die Unterversorgung in diesem Bereich etwas abschwächte. ${ }^{77}$ Der militärische Bedarf konnte während des Kriegs insgesamt aber ,glatt gedeckt“ werden. ${ }^{78}$

71 Für nähere Ausführungen zu den beiden Werken siehe Fehr, Vulnerabilität, S. 38-42.

72 BAR, E 7350 1000/1104, Bd. 42 (q), Rapport über die Beschaffung von Salpetersäure für die schweizerische Industrie o.J., S. 2.

73 Geering, Handel, S. 700.

74 Abteilung für industrielle Kriegswirtschaft, Abteilung, S. 40, 48-49.

75 BAR, E 7350 1000/1104, Bd. 42 (q), Rapport über die Beschaffung von Salpetersäure für die schweizerische Industrie o.J., S. 1.

76 Geering, Handel, S. 700.

77 Fehr, Vulnerabilität, S. 38.

78 Waeser, Luftstickstoff-Industrie, S. 118. 


\section{Schweizer Unternehmen als Zulieferer der deutschen Stickstoffwirtschaft}

Die Versorgung der Schweiz mit Stickstoffverbindungen ist für die Untersuchung der Rolle der Schweizer Unternehmen in der deutschen Stickstoffwirtschaft insofern von Bedeutung, als auf dieser Grundlage abgeschätzt werden kann, welche Stoffe und Ressourcen die Schweiz grundsätzlich auszuführen imstande war. Wie bereits dargelegt wurde, können nennenswerte Ausfuhren von Chilesalpeter nach Deutschland zur Umgehung der Blockade weitgehend ausgeschlossen werden, da es der Schweiz an diesem Rohstoff selber stark mangelte. Auch andere Stickstoffverbindungen, wie Kalkstickstoff oder Ammoniak, kann Deutschland nicht indirekt über die Schweiz bezogen haben, weil diese selbst praktisch gar keine derartigen Substanzen einführte. Zudem unterlagen die Einfuhren in die Schweiz aus den Ländern der Entente der Kontrolle der Überwachungsgesellschaft Société suisse de surveillance économique (S.S.S.), die Wiederausfuhren relativ erfolgreich unterband. Praktisch keinen Einfluss hatte diese Gesellschaft aber auf die Ausfuhr von in der Schweiz selber hergestellten Gütern, bei deren Erzeugung keine von der Entente gelieferten Rohstoffe genutzt wurden. ${ }^{79}$ Solange die Schweiz keine der Kriegsparteien einseitig begünstigte, waren derartige Ausfuhren auch neutralitätspolitisch unbedenklich. ${ }^{80}$

Die Unternehmen waren bei der Ausfuhr ihrer Erzeugnisse aber dennoch Einschränkungen unterworfen. Der Schweizerische Bundesrat verhängte nämlich bereits kurz nach Kriegsbeginn weitreichende Ausfuhrverbote für praktisch alle Arten von Stickstoffverbindungen: im August 1914 für Chilesalpeter, Salpetersäure und Explosivstoffe, im September 1914 für alle Arten von Salpeter und sämtliche Kunstdünger, im November 1914 für Stickoxide und salpetersaure Salze und 1915 auch für Ammoniak. ${ }^{81} \mathrm{Zu}$ den während des Kriegs erlassenen Ausfuhr-

79 Fehr, Stickstofffrage, S. 122-126.

80 Gemäß Art. 7 bis 9 des Haager Abkommens betreffend die Rechte und Pflichten der neutralen Mächte und Personen im Falle eines Landkriegs. (Schweizerischer Bundesrat, Botschaft des Bundesrates an die Bundesversammlung betreffend die Ergebnisse der im Jahre 1907 im Haag abgehaltenen zweiten internationalen Friedenskonferenz vom 28. Dezember 1908, in: Schweizerisches Bundesblatt 1, 1909, S. 257. Siehe auch J. Ruchti, Geschichte der Schweiz während des Weltkrieges, 1914-1919. Politisch, wirtschaftlich und kulturell 2, Kriegswirtschaft und Kulturelles, Bern 1930, S. 34-35.)

81 Bundesratsbeschluss betreffend Ausfuhrverbote vom 13. August 1914, in: Amtliche Sammlung der Bundesgesetze und Verordnungen der Schweizerischen Eidgenossenschaft 1914, S. 384-385; Bundesratsbeschluss betreffend Ausfuhrverbote vom 18. September 1914, in: Amtliche Sammlung der Bundesgesetze und Verordnungen der Schweizerischen Eidgenossenschaft 1914, S. 483- 
verboten ist es allerdings wichtig $\mathrm{zu}$ verstehen, dass diese - entgegen ihres Wortlauts - keineswegs eine vollständige Einstellung der Exporte zum Ziel, sondern lediglich eine „exportregelnde Funktion“ hatten. Die „Verbote“ ermöglichten es dem Staat, den Export mittels der Vergabe von Ausnahmebewilligungen zu kontrollieren und zu steuern. Dies war nicht zuletzt auch im Rahmen des regen Kompensationshandels der Schweiz mit den benachbarten kriegführenden Staaten von großer Bedeutung. ${ }^{82}$

Im Fall der kriegswirtschaftlich besonders wichtigen Stickstoffverbindung Salpetersäure wurde bereits ausgeführt, dass die beiden Firmen Nitrum AG und AIAG in den ersten beiden Kriegsjahren auch Exporte tätigen durften. Eine Aufschlüsselung nach Empfängerstaaten zeigt, dass die Ausfuhren im Umfang von 679 Tonnen Salpetersäure 1914 praktisch vollständig nach Deutschland gingen, während 1915 von den insgesamt 661 ausgeführten Tonnen immerhin 545 Tonnen bzw. 82 Prozent der deutschen Kriegswirtschaft zugutekamen. 1916 setzte, wie bereits erwähnt, eine Beschlagnahmung sämtlicher Schweizer Salpetersäure für die schweizerische Munitionserzeugung ein, weshalb in diesem Jahr nur noch vernachlässigbare 11 Tonnen ausgeführt wurden, die vollständig Deutschland zugutekamen. Für die Jahre 1917 und 1918 weist die Schweizer Zollstatistik gar keine Ausfuhren von Salpetersäure mehr aus. ${ }^{83}$ Tatsächlich sind aber auch nach der allgemeinen Beschlagnahmung weiterhin bescheidene, in den Statistiken nicht deklarierte Exporte nach Deutschland erfolgt. Dies belegen Quellen im Deutschen und im Schweizerischen Bundesarchiv, denen entnommen werden kann, dass die Schweiz auch in den Jahren 1917 und 1918 Salpetersäure im Umfang von mehreren Dutzend Tonnen nach Deutschland lieferte, um damit kriegswichtige Importe aus Deutschland, so beispielsweise von Sprengkapseln oder von anderen Stickstoffverbindungen, zu kompensieren. ${ }^{84}$

Weitere Salpetersäureexporte nach Deutschland erfolgten in diesen Jahren zur Kompensation von Lieferungen deutscher Bauteile und Chemikalien, die für

\footnotetext{
484; Bundesratsbeschluss betreffend Ausfuhrverbote vom 5. November 1915, in: Amtliche Sammlung der Bundesgesetze und Verordnungen der Schweizerischen Eidgenossenschaft 1915, S. 357-360; Bundesratsbeschluss betreffend die Ausdehnung der Ausfuhrverbote vom 18. September, 20. und 27. Oktober 1914, vom 27. November 1914, in: Amtliche Sammlung der Bundesgesetze und Verordnungen der Schweizerischen Eidgenossenschaft 1914, S. 584-585.

82 H. Sieveking, Schweizerische Kriegswirtschaft, Lausanne 1922, S. 12. Siehe auch Roth, Staat, S. 257-258.

83 Abteilung für industrielle Kriegswirtschaft, Abteilung, S. 49.

84 BArch, R 8729 288, Schreiben des Waffen- und Munitionsbeschaffungs-Amts an die KriegsChemikalien-Aktiengesellschaft vom 07.02.1917; BAR, E 27 18683, Bd. 2, Schreiben des Chefs des Generalstabs der Schweizer Armee an den Militärattaché bei der Kaiserlich Deutschen Gesandtschaft in Bern vom 04.10.1918.
} 
den Betrieb des Werks der Nitrum AG in Bodio zwingend erforderlich waren. Wie sich zeigte, konnte diese Fabrik trotz der Aufspaltung des Unternehmens in einen schweizerischen und einen deutschen Teil im Jahr 1915 nicht ohne Unterstützung aus Deutschland betrieben werden. In einem Rapport des Politischen Departements der Schweizerischen Eidgenossenschaft hieß es dazu: „Ob also Bodio läuft oder nicht ist ganz vom guten Willen der elektrochemischen Werke Bitterfeld und der deutschen Regierung abhängig.“ Man könne daher „mit dieser Fabrik für die Landesverteidigung bei der geringsten Komplikation mit Deutschland überhaupt nicht rechnen“. ${ }^{85}$ Das einzige Salpetersäurewerk, das in der Schweiz autark betrieben werden konnte, war dasjenige der AIAG in Chippis. ${ }^{86}$ Wie gefährlich die fehlende Redundanz und die damit verbundene Verletzlichkeit der schweizerischen Salpetersäureversorgung war, zeigte sich am 1. Mai 1917, als das Werk Chippis der AIAG bei einem Sprengstoffanschlag nur um Haaresbreite der Zerstörung entging. ${ }^{87}$ Der Angriff auf die „Achillesferse“ der schweizerischen Explosivstofferzeugung verursachte in der Führung der Schweizer Armee große Aufregung und hatte unter anderem die sofortige militärische Bewachung der Anlagen zur Folge. ${ }^{88}$ Obschon die genauen Hintergründe und die Täterschaft bis heute nicht restlos geklärt sind, wird in der Literatur davon ausgegangen, dass der Angriff nicht der schweizerischen Salpetersäureproduktion, sondern der auf demselben Werkareal gelegenen, ebenfalls äußerst energieintensiven Aluminiumerzeugung gegolten habe, die überwiegend der deutschen Kriegswirtschaft zugutekam. Als Täter werden „Agenten des französischen Spionagebüros Evian/Thonon" vermutet. ${ }^{89}$

Größer als bei der Salpetersäure war der Produktionsüberschuss der schweizerischen chemischen Industrie im Bereich der Kalkstickstofferzeugung in den Werken Gampel der Lonza AG und Martigny der Société des Produits Azotés. Diese exportierten während des gesamten Kriegs den größten Teil ihrer Produktion. Insgesamt waren dies im Jahr 1914 5.500 Tonnen und in den nachfolgenden

85 BAR, E 7350 1000/1104, Bd. 42 (q), Rapport über die Beschaffung von Salpetersäure für die schweizerische Industrie o.J., S. 1-4.

86 Fehr, Vulnerabilität, S. 39-40.

87 BAR, E 21 14412, Bericht über das Attentat auf die Druckwasserleitung der Aluminium Industrie AG in Chippis bei Siders (Wallis) vom 03.05.1917, S. 1-5.

88 Siehe dazu ausführlich Fehr, Vulnerabilität, S. 42.

89 F. Garbely, Kanton Alusuisse. Alusuisse im Wallis, in: T. Bauer/G.J. Crough/E. Davidsson/F. Garbely/P. Indermaur/L. Vogel (Hg.), Silbersonne am Horizont. Alusuisse - Eine Schweizer Kolonialgeschichte, Zürich 1989, S. 199; B. Aymon, Un demi-siècle de lutte ouvrière à l'aluminium de Chippis, Genève 1979, S. 33; A. Schweri, La grève de 1917 aux usines d'aluminium de Chippis. Un exemple de traumatisme industriel en pays agricole, Genève 1988, S. 93. 
Jahren 11.699 Tonnen (1915), 20.021 Tonnen (1916), 16.701 Tonnen (1917) und 13.438 Tonnen (1918). Davon gingen total rund 46 Prozent an die Mittelmächte und unter diesen primär an Deutschland sowie 54 Prozent an die Entente. ${ }^{90}$ Was den Verwendungszweck des Kalkstickstoffs in den Empfängerländern betrifft, war allen Beteiligten bewusst, dass er nicht etwa als Dünger auf die Äcker gestreut, sondern in der Stickstoffindustrie zu Salpetersäure weiterverarbeitet wurde und diese wiederum der Explosivstoff- bzw. der Munitionserzeugung diente. ${ }^{91}$

Eine dritte Stickstoffverbindung, die während des Kriegs in nennenswertem Umfang aus der Schweiz exportiert wurde, war das in den Gaswerken mittels Trockendestillation von Kohle gewonnene Ammoniak. Weil in der Schweiz während des Kriegs nur verschwindend kleine Mengen qualitativ unzureichender Kohle gefördert wurden, war die Kohleversorgung des Landes praktisch vollständig von ausländischen Zulieferern abhängig. ${ }^{92}$ Somit handelte es sich bei dem in den Gaswerken anfallenden Ammoniak nur bedingt um ein Schweizer Produkt. Da während des Kriegs der überwiegende Teil der in der Schweiz verwendeten Kohle aus Deutschland stammte, ist es nicht weiter verwunderlich, dass auch die Ammoniakausfuhren aus der Schweiz mehrheitlich Deutschland zugutekamen. ${ }^{93}$ Offensichtlich musste der Schweizerische Bundesrat im Gegenzug für die deutschen Kohlelieferungen sogar eine Garantie abgeben, „die bei der Gasfabrikation anfallenden Nebenprodukte (Ammoniak oder Teer) [...], soweit sie nicht in der Schweiz verbraucht würden, nach Deutschland“ auszuführen. ${ }^{94}$ Insgesamt dürfte es sich bei diesen Exporten während des gesamten Kriegs um knapp unter 1.000 Tonnen Ammoniak gehandelt haben. ${ }^{95}$

Um die Exporte der verschiedenen Stickstoffverbindungen nach Deutschland quantitativ vergleichen und deren Bedeutung für die deutsche Stickstoffwirtschaft beurteilen zu können, bietet sich die branchenübliche Umrechnung der Gewichtsangaben auf ihren Stickstoffgehalt an. Dabei zeigt sich, dass die Ausfuhr in den Jahren 1914 bis 1916 jeweils rund 2.000 Jahrestonnen Stickstoff betrug und sich in den letzten beiden Kriegsjahren auf rund 1.000 Jahrestonnen Stickstoff halbierte. Insgesamt hatte das Ammoniak an diesen Ausfuhren ledig-

90 Abteilung für industrielle Kriegswirtschaft, Abteilung, S. 58.

91 Lonza AG (Hg.), 50 Jahre, S. 21; C. Fux, 100 Jahre Lonza-Produkte. Von der Carbidlampe zur Feinchemie, 1897-1997, in: Aktuell. Zeitschrift der Mitarbeiterinnen und Mitarbeiter der Lonza AG. Sonderausgabe zum 100-jährigen Bestehen der Lonza, 1997, S. 30.

92 Geering, Handel, S. 112-116.

93 Ebda., S. 93; Abteilung für industrielle Kriegswirtschaft, Abteilung, S. 55.

94 H. Ochsenbein, Die verlorene Wirtschaftsfreiheit, 1914-1918. Methoden ausländischer Wirtschaftskontrollen über die Schweiz, Bern 1971, S. 194.

95 Abteilung für industrielle Kriegswirtschaft, Abteilung, S. 54. 
lich einen Anteil von 8 Prozent, während die Salpetersäure sogar nur 3 Prozent ausmachte. Die mit 89 Prozent größte Stickstoffausfuhr erfolgte in Form von Kalkstickstoff (siehe Abb. 2). ${ }^{96}$ Obschon diese Lieferungen aufgrund des Mangels in Deutschland höchst willkommen waren, darf ihre Bedeutung für die deutsche Versorgung nicht überschätzt werden. Im Vergleich mit der Produktion der deutschen Stickstoffwirtschaft zeigt sich nämlich, dass die aus der Schweiz stammenden Mengen nie mehr als zwei Prozent der deutschen Gesamterzeugung ausmachten und somit als geringfügig zu bezeichnen sind. ${ }^{97}$ Dennoch kann zusammenfassend festgestellt werden, dass Schweizer Unternehmen die in den Alpen verfügbare Wasserkraft für den Betrieb äußerst energieintensiver Verfahren zur Stickstofffixierung nutzten und die gewonnenen Stickstoffverbindungen während des Ersten Weltkriegs auch nach Deutschland ausführten, wo sie der Explosivstoff- und Munitionserzeugung dienten. ${ }^{98}$

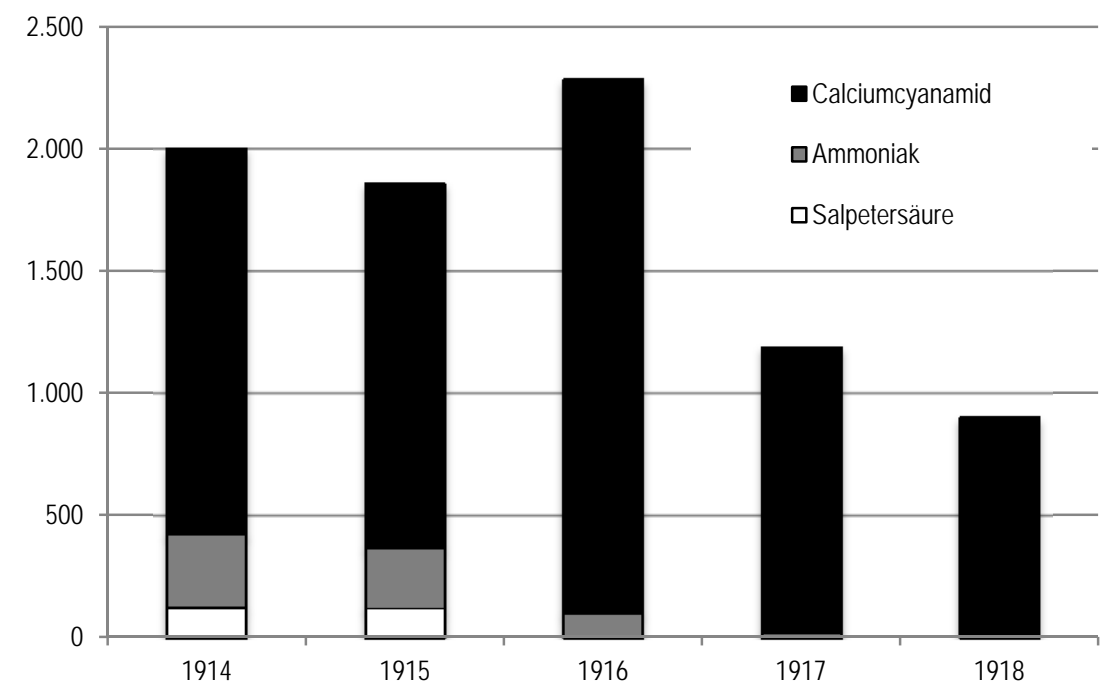

Abb. 2: Die Ausfuhr von Stickstoffverbindungen aus der Schweiz nach Deutschland 1914-1918 in Tonnen Stickstoff ${ }^{99}$

96 Fehr, Stickstofffrage, S. 135, 176, 181.

97 Ebda., S. 134.

98 Ebda., S. 132.

99 Ebda., S. 181. 
Eine Untersuchung der Rolle schweizerischer Unternehmen als Zulieferer in der deutschen Stickstoffwirtschaft darf sich nicht auf Stickstoffverbindungen beschränken, sondern soll auch Zwischenerzeugnisse und Ressourcen berücksichtigen, die für die Produktion von Stickstoffverbindungen erforderlich sind. Wie bereits ausgeführt, wandelte sich die ursprüngliche, globale Stickstofffrage mit der praktischen Umsetzung chemieindustrieller Verfahren zur Fixierung des Stickstoffs der Luft zu einer Energiefrage. Im Folgenden soll daher die Lieferung von Energie und energieintensiven Zwischenerzeugnissen im Vordergrund stehen. Von Bedeutung ist die Untersuchung der Produktionskette insbesondere bei der Herstellung von Kalkstickstoff, da der Prozess der Azotierung bzw. der Bindung des Stickstoffs bei diesem Verfahren wie bereits erwähnt exotherm, d.h. unter Abgabe von Energie, verläuft. Das eigentliche Schlüsselelement der Produktionskette ist allerdings nicht diese Reaktion, sondern die Herstellung des dazu erforderlichen Calciumcarbids. Dieses muss unter erheblichem Energieaufwand im elektrischen Ofen erzeugt werden und ist auch der Grund dafür, dass die Kalkstickstoffproduktion insgesamt sehr viel Energie benötigt. Da die beiden Teilprozesse nicht zwingend am selben Produktionsstandort vollzogen werden müssen, soll auch untersucht werden, ob Schweizer Unternehmen die deutschen Kalkstickstoffwerke mit Calciumcarbid belieferten.

Aufgrund der verfügbaren günstigen Wasserkraft etablierte sich in der Schweiz bereits vor dem Ersten Weltkrieg eine ausgedehnte Karbidindustrie. Bei Kriegsbeginn betrieb die Lonza AG je ein Karbidwerk in Gampel und in Thusis, Gregor Stächelin eines in Vernayaz, P. Spörri eines in Flums, Gustav Weinmann je eines in Chavornay und Kallnach, die Société Anonyme Electrométallurgique Procédés P. Girod eines in Courtepin und die Société des Produits Electrométallurgiques SA ein weiteres Werk in Montbovon. Diese acht Fabriken erzeugten 1913 bereits 46.598 Tonnen Karbid, wovon 31.790 Tonnen zur Ausfuhr gelangten, von denen wiederum 25.013 Tonnen bzw. rund 80 Prozent an Deutschland geliefert wurden. ${ }^{100}$ Calciumcarbid war also bereits vor dem Krieg ein echter Exportschlager der Schweiz. Als Grund für den Erfolg erkannte die damalige Industrie die Funktion des Karbids als Speicher von Energie: „Das Calciumcarbid kann man zur Zeit als den verbreitetsten und einfachsten Akkumulator der elektrischen Energie betrachten, durch dessen Vermittlung große Energiemengen unserer Gebirgswasser aufgespeichert [...] werden." ${ }^{101}$

100 Abteilung für industrielle Kriegswirtschaft, Abteilung, S. 100-101.

101 Lonza AG (Hg.), Elektrizitätswerk Lonza. Aktiengesellschaft mit Sitz in Gampel (Wallis). Verwaltung in Basel, o.O.u.J., S. 32. 
Im Jahr 1914 brach die Karbidproduktion zwar zunächst leicht ein, setzte anschließend aber zu einem bemerkenswerten Wachstum an, als nicht nur mindestens zehn weitere Werke gegründet wurden, sondern sich auch die Produktion auf über 100.000 Tonnen Calciumcarbid im Jahr 1918 mehr als verdoppelte. Die neuen Werke entstanden unter anderem in Aarau (Société d'Electrochimie d'Aarau), Martigny (Société des Produits azotés), Vallée du Day (Société des Carbures du Day), Vouvry (Société Romande d'Electricité Territet), Visp (Lonza AG), Meiringen (Elektrowerke Reichenbach AG) und Buchs (Société Electrothermique de Buchs). In Bodio in den Tessiner Alpen, wo besonders große Mengen von Wasserkraft verfügbar waren, errichteten neben dem im vorliegenden Artikel bereits beschriebenen, nach dem Lichtbogenverfahren produzierenden Salpetersäurewerk der Nitrum AG gleich drei Unternehmen neue Karbidfabriken. Es handelte sich dabei um die Société des Carbures du Day, die Société Electrochimique du Léman SA sowie die Gotthardwerke AG für elektrochemische Industrie. Insgesamt produzierten die schweizerischen Unternehmen in den Jahren 1914 bis 1918 rund 360.000 Tonnen Calciumcarbid, wovon nicht einmal 87.000 Tonnen bzw. 24 Prozent im Inland verwendet wurden. ${ }^{102}$ Der mit Abstand größte Teil der Produktion von rund 206.000 Tonnen bzw. 57 Prozent wurde stattdessen nach Deutschland exportiert, während die Entente weniger als 17 Prozent erhielt (siehe Abb. 3). ${ }^{103}$

Die Höhe der deutschen Bezüge dürfte sich teilweise damit erklären lassen, dass die deutsche Wirtschaft bereits vor dem Krieg der wichtigste Exportkunde der Schweiz war. Nach dem Kriegsbeginn unterlag Calciumcarbid zunächst keinen Ausfuhrbeschränkungen. Erst im März 1916 verhängte der Schweizerische Bundesrat auch über diese Ressource ein Ausfuhrverbot, um sich eine Einflussnahme auf die Exporte zu ermöglichen. ${ }^{104}$ Deutschland blieb aber auch anschließend der größte Abnehmer des schweizerischen Karbids. Im Anhang des deutsch-schweizerischen Handelsabkommens vom 2. September 1916 wurde in

102 Abteilung für industrielle Kriegswirtschaft, Abteilung, S. 111-113.

103 Schweizerisches Zolldepartement (Hg.), Statistik des Warenverkehrs der Schweiz mit dem Auslande 1914, Bern 1915, S. 169; Dass., Statistik des Warenverkehrs der Schweiz mit dem Auslande 1915, Bern 1916, S. 161; Dass., Statistik des Warenverkehrs der Schweiz mit dem Auslande 1916, Bern 1917, S. 160; Dass., Statistik des Warenverkehrs der Schweiz mit dem Auslande 1917, Bern 1918, S. 151; Dass., Statistik des Warenverkehrs der Schweiz mit dem Auslande 1918, Bern 1919, S. 142; Abteilung für industrielle Kriegswirtschaft, Abteilung, S. 112.

104 Bundesratsbeschluss betreffend Ausfuhrverbote vom 6. März 1916, in: Amtliche Sammlung der Bundesgesetze und Verordnungen der Schweizerischen Eidgenossenschaft 1916, S. 7172; BAR, E 7350 1000/1104, Bd. 39-40 (f), Schreiben der Sektion Chemie der Abteilung für industrielle Kriegswirtschaft an das Bezirksgericht Zürich vom 20.12.1917, S. 1. 
einem Sonderabkommen sogar verbindlich festgehalten, dass die Schweiz zur Kompensation von deutschen Kohle- und Stahllieferungen weiterhin Calciumcarbid nach Deutschland exportieren und auch für die Einhaltung einer Reihe von Lieferverträgen der deutschen Kriegschemikalien AG und der Kriegsmetall AG mit schweizerischen Karbidfabriken sorgen werde. Die Reichsregierung sicherte der Schweiz im Gegenzug zudem die für die Produktion des Karbids erforderlichen „Rohstoffe, Materialien und Apparate“ zu. ${ }^{105}$

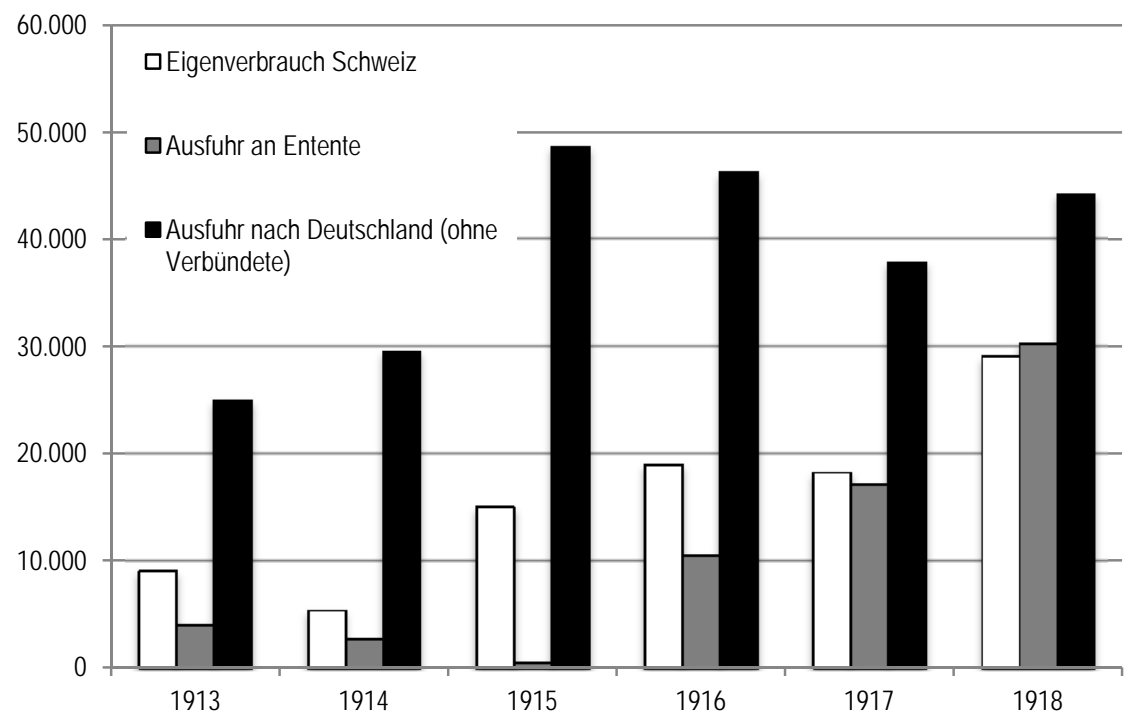

Abb. 3: Die Ausfuhr von Calciumcarbid aus der Schweiz nach Deutschland, an die Entente und der Schweizer Eigenverbrauch 1913-1918 (in Tonnen) ${ }^{106}$

Die Verwendung des nach Deutschland ausgeführten Calciumcarbids kann nicht mehr im Detail rekonstruiert werden, da bei der Ausfuhr keine entsprechende Unterscheidung vorgenommen wurde und die Verteilung in Deutschland oftmals dezentral in sogenannten Verkaufsgenossenschaften erfolgte. ${ }^{107}$ Nebst der Kalkstickstofffabrikation wurde Calciumcarbid auch für die Gewinnung von

105 BAR, E 7350 (-) -/1, Bd. 5, Deutsch-Schweizerisches Abkommen vom September 1916, Anlage I, Sonder-Abkommen über Hüttenaluminium, Aluminium-Legierungen und Produkte des elektrischen Ofens, sowie elektrische Energie, S. 1-2.

106 Fehr, Stickstofffrage, S. 181-182.

107 BAR, E 7350 1000/1104, Bd. 39-40 (f), Protokoll über die Carbidsitzung vom 21.08.1916, S. 2; BAR, E 7350 1000/1104, Bd. 39-40 (f), Protokoll über die Carbidsitzung vom 22.08.1916, S. 1. 
Acetylengas verwendet, das zur Beleuchtung, zum Schweißen, zum Betrieb von Automobilen und als Ausgangsstoff der Acetylenchemie diente. Ein weiterer kriegswirtschaftlich höchst bedeutsamer Verwendungszweck von Karbid war die Entschwefelung von Eisen in der Stahlindustrie. ${ }^{108}$ Obschon das schweizerische Calciumcarbid in der deutschen Wirtschaft zweifellos in verschiedensten Bereichen verwendet wurde, kann dennoch einwandfrei belegt werden, dass zumindest ein Teil der deutschen Kalkstickstoffindustrie zugeführt wurde. ${ }^{109} \mathrm{Zu}$ den in den Quellen explizit genannten Kunden zählen neben den Bayerischen Stickstoffwerken in Trostberg auch die AG für Stickstoffdünger in Knapsack. ${ }^{110}$ Auch die französische Botschaft in Bern war davon überzeugt, dass das Schweizer Karbid in Deutschland zu Kalkstickstoff und dieser wiederum zur Produktion von Explosivstoffen bzw. von Munition herangezogen würde, was sie beim Schweizerischen Bundesrat beanstandete. ${ }^{111}$

Ausgehend von einer Schätzung Bruno Waesers, wonach in Deutschland gegen Ende des Kriegs rund 74 Prozent des insgesamt zur Verfügung stehenden Calciumcarbids für die Kalkstickstofffabrikation und lediglich 26 Prozent für andere Zwecke, wie Beleuchtung, Metallverarbeitung etc. verwendet wurden, kann dennoch grob abgeschätzt werden, wie stark das schweizerische Karbid zur deutschen Kalkstickstofferzeugung beigetragen hat. ${ }^{112}$ Unter der Annahme, dass die oben genannten 74 Prozent auch für die Importe aus der Schweiz zutreffen, kann errechnet werden, dass von den zwischen 1914 und 1918 insgesamt aus der Schweiz bezogenen 206.000 Tonnen Karbid rund 152.000 Tonnen der deutschen Kalkstickstoffindustrie zugeführt wurden, welche daraus rund 204.000 Tonnen Calciumcyanamid hätte herstellen können, was rund 41.000 Tonnen gebundenen Stickstoffs oder über 30 Prozent der gesamtem deutschen Kalkstickstofferzeugung der Jahre 1914 bis 1918 entspricht. Diese Schätzung zeigt deutlich, dass die Lieferung des Zwischenerzeugnisses Calciumcarbid, im Gegensatz zu den Schweizer Lieferungen primärer Stickstoffverbindungen, eine beträchtliche Stützung

108 F. Winteler, Die heutige industrielle Elektrochemie. Ein Überblick mit besonderer Berücksichtigung der schweizerischen Verhältnisse, Zürich 1919, S. 21.

109 Siehe dazu ausführlich Fehr, Stickstofffrage, S. 138-141.

110 BArch, R 8729 338, Schreiben der Kaiserlich Deutschen Gesandtschaft in Bern an die Kriegschemikalien AG vom 30. Mai 1917; BArch, R 8729 340, Vertrag zwischen der Kriegsmetall AG und der Lonza AG vom 17.11.1916, S. 3.

111 BAR, E 2001 (A) 897, Schreiben der französischen Botschaft in Bern an den Chef des Politischen Departements, Bundesrat Hoffmann vom 06.06.1917, S. 2.

112 Waeser, Luftstickstoff-Industrie, S. 27. 
der deutschen Kalkstickstoffindustrie bzw. der deutschen Stickstoffwirtschaft darstellte. ${ }^{13}$

Die in der Schweiz aus Wasserkraft gewonnene Energie wurde der deutschen Stickstoffwirtschaft nicht nur indirekt mittels Exporten von Salpetersäure, Calciumcyanamid oder Calciumcarbid, sondern auch viel direkter, in Form elektrischer Energie zugeführt. Im Gegensatz zu den Exporten der genannten Substanzen war die Ausfuhr von Elektrizität aus der Schweiz nicht erst aufgrund des Ersten Weltkriegs, sondern bereits seit 1906 bewilligungspflichtig. ${ }^{114}$ In der Praxis lehnte der Schweizerische Bundesrat aber kaum Gesuche ab, weshalb bereits vor dem Ersten Weltkrieg zahlreiche Elektrizitätsausfuhren bewilligt worden waren. ${ }^{115}$

Auch für die im deutschen Waldshut gelegene Lonza-Werke elektrochemische Fabriken $\mathrm{GmbH}$, die eine Tochtergesellschaft der schweizerischen Lonza AG war und ab 1913 Calciumcarbid sowie ab 1916 Calciumcyanamid herstellte, bewilligte der Schweizerische Bundesrat 1913 eine erste Elektrizitätsausfuhr von 12.000 Kilowatt. Während des Kriegs folgten weitere Bewilligungen für 10.000 Kilowatt (1915), 5.000 Kilowatt (1916) und 12.000 Kilowatt (1917). Die Bewilligungen wurden der AG „Motor“ in Baden und der Nordostschweizerischen Kraftwerke AG erteilt, welche die Elektrizität in den Kraftwerken Laufenburg, Beznau und Olten-Gösgen produzierten. ${ }^{116}$

Ein weiteres deutsches Stickstoffwerk, das mit Elektrizität aus einem Rheinkraftwerk betrieben wurde, war die mit dem Lichtbogenverfahren produzierende Elektro-Nitrum AG in Rhina, die vom Kraftwerk Laufenburg versorgt wurde. ${ }^{117}$ Da es sich bei diesem jedoch um ein deutsch-schweizerisches Grenzkraftwerk handelte, das je zur Hälfte nach Deutschland und in die Schweiz lieferte, und die Elektro-Nitrum AG ihre Elektrizität aus der deutschen Produktionshälfte be-

113 Fehr, Stickstofffrage, S. 140-141, 180-182.

114 Bundesbeschluss über die Abgabe inländischer Wasserkräfte ins Ausland vom 31. März 1906, in: Amtliche Sammlung der Bundesgesetze und Verordnungen der Schweizerischen Eidgenossenschaft 1916, S. 160.

115 H. Trümpy, Ausfuhr elektrischer Energie, Zürich 1925, S. 14; Schweizerischer Bundesrat, Bericht des schweizerischen Bundesrates an die Bundesversammlung über seine Geschäftsführung im Jahre 1913, Bern 1914, S. 696.

116 Ebda.; Ders., Bericht des schweizerischen Bundesrates an die Bundesversammlung über seine Geschäftsführung im Jahre 1915, Bern 1916, S. 247; Ders., Bericht des schweizerischen Bundesrates an die Bundesversammlung über seine Geschäftsführung im Jahre 1916, Bern 1917, S. 171; Ders., Bericht des schweizerischen Bundesrates an die Bundesversammlung über seine Geschäftsführung im Jahre 1917, Bern 1918, S. 198.

117 W. Bocks, Pioniergeist. 100 Jahre Wasserkraft aus Laufenburg. 100 Jahre Energie vom Hochrhein, Laufenburg 2008, S. 64, 68; J. Rennhard/K. Malleis, 75 Jahre Kraftwerk Laufenburg, Laufenburg 1983, S. 47-48; A. Steigmeier, Power on. Elektrowatt, 1895-1995, Zürich 1995, S. 43. 
zog, handelte es sich bei diesem Strombezug nicht um einen Export aus der Schweiz. ${ }^{118}$ Wie wichtig die Rheinkraftwerke für die deutsche Kriegswirtschaft waren, zeigt der Umstand, dass der deutsche Generalstab den schweizerischen Generalstab im September 1916 vor möglichen Sprengstoffanschlägen der Entente auf die gemeinsamen Stauanlagen warnte. ${ }^{119}$ Wie berechtigt diese Sorge war, zeigte sich am 16. Oktober, als Unbekannte das deutsch-schweizerische Grenzkraftwerk Rheinfelden nachts mit der Zündung von drei Sprengsätzen erfolglos zu beschädigen versuchten und ein Schweizer Grenzwächter am selben Tag weitere 23 unbenutzte „Schwimmbomben“ auf einem am Schweizer Rheinufer liegenden Boot entdeckte. ${ }^{120}$ Das von diesem Kraftwerk mit elektrischer Energie versorgte, ebenfalls im deutschen Rheinfelden liegende Werk der chemischen Fabrik Griesheim-Elektron produzierte zwar keine Stickstoffverbindungen. ${ }^{121}$ Der Sprengstoffanschlag zeigt aber dennoch, dass die hinter dem Anschlag vermutete Entente die Energieversorgung der deutschen chemischen Industrie durch die deutsch-schweizerischen Rheinkraftwerke als derart relevant einstufte, dass sie deren Beschädigung oder gar Zerstörung anstrebte. Die Schweizer Armee stellte daher neben anderen Rheinkraftwerken auch den Schweizer Teil des Kraftwerks Laufenburg, welches das deutsche Salpetersäurewerk der ElektroNitrum AG mit Elektrizität versorgte, unter militärische Bewachung. ${ }^{122}$ Der Regierungsrat des Kantons Aargaus meinte dazu: „Die Werke haben internationalen Charakter. Hieraus entsteht für die schweizerischen Behörden die Pflicht, auch mit Rücksicht auf die deutschen Interessen, alles zu tun, um Schädigungen der Werkanlagen zu verhüten.“123

118 BArch, R 4604 226, Schreiben der Kraftwerk Reckingen AG an das Hauptzollamt Waldshut vom 08.07.1938, S. 3 .

119 BAR, E 21 14400, Schreiben des Stabschefs der 6. Division an den Generalstabschef der schweizerischen Armee vom 22.09.1916, S. 1.

120 BAR, E 21 14400, Schreiben der Kraftübertragungswerke Rheinfelden an die II. Kompagnie des Landsturm Natl. XIV/19 vom 17.10.1916, S. 1; BAR, E 21 14400, Schreiben des Bezirksamtes Rheinfelden über den Bombenanschlag auf das Kraftwerk Rheinfelden vom 17. Oktober 1916, S. 1-3; BAR, E 21 14400, Schreiben des Regierungsrates des Kantons Aargau an den Schweizerischen Bundesrat vom 19.10.1916, S. 1.

121 G. Pistor, Hundert Jahre Griesheim, 1856-1956. Ein Beitrag zur Geschichte der chemischen Industrie, Tegernsee 1958, S. 46, 219; P. Horster, Die volkswirtschaftliche Bedeutung der chemischen Industrie am Oberrhein, besonders als elektrolytische, elektrothermische und metallurgische Industrie. Ein Beitrag zur Industrialisierung durch Wasserkräfte, Basel 1922, S. 12.

122 BAR, E 21 14400, Schreiben des Kommandos der Guiden-Brigade an die Nachrichtensektion des Armeestabs vom 21.10.1916, S. 1.

123 BAR, E 21 14400, Schreiben des Regierungsrates des Kantons Aargau an den Schweizerischen Bundesrat vom 19.10.1916, S. 2. 


\section{Schweizer Unternehmen als Produzenten in der deutschen Stickstoffwirtschaft}

Zur Stärkung ihrer Marktposition in Deutschland gründete die Lonza AG im Herbst 1913 in Waldshut, unmittelbar auf der deutschen Seite des Rheins, ihre erste Tochtergesellschaft Lonza-Werke elektrochemische Fabriken GmbH.124 Diese sollte auf der Grundlage der bereits erwähnten Elektrizitätsimporte aus der Schweiz die Produktion von Calciumcarbid aufnehmen. ${ }^{125}$ Der Beginn des Ersten Weltkriegs erschwerte die 1914 aufgenommenen Bauarbeiten stark, da die Mehrheit der deutschen Arbeiter in den Militärdienst einberufen wurde. Diese wurde in der Folge „fast ausschließlich“ durch Schweizer Arbeitskräfte ersetzt. ${ }^{126} \mathrm{Im}$ Jahr 1915 liefen die Anlagen zur Herstellung von Calciumcarbid erstmals an. ${ }^{127}$ Auch bei der Produktion setzte das Werk neben Kriegsgefangenen und dienstverpflichteten Deutschen auch Schweizer Arbeitskräfte ein, die über die unmittelbar neben dem Werk befindliche Rheingrenze nach Waldshut pendelten. Die Nutzung schweizerischer Ressourcen durch dieses, sich auf deutschem Boden befindliche und für die deutsche Kriegswirtschaft produzierende Werk ging jedoch weit über die Elektrizität und die Arbeitskräfte hinaus. So wurde auch der für die Karbiderzeugung erforderliche Kalk aus der Schweiz bezogen. Um diese Bezüge möglichst effizient zu gestalten, verlegten die Schweizer Behörden auch die Grenzkontrollen für die Kalktransporte auf das Gelände der Lonza-Werke im deutschen Waldshut. ${ }^{128}$

Zur Optimierung der Nutzung schweizerischer Ressourcen beschloss die Lonza sogar, ihre in Waldshut eingesetzten Schweizer Arbeitskräfte jeweils auf der schweizerischen Seite des Rheins mit Schweizer Lebensmitteln zu verpflegen. Dazu wurde eigens eine Kantine errichtet und die Arbeiter jeweils mit einer Rheinfähre über die Grenze befördert. Es ist überliefert, dass die Schweizer Arbeiter für ihre unter der schlechten Ernährungslage leidenden deutschen Arbeitskollegen gelegentlich auch Lebensmittel über die Grenze schmuggelten. In einem Fall soll ein Schweizer Lonza-Arbeiter dabei ertappt worden sein, wie er

124 Lonza AG (Hg.), 75 Jahre Lonza AG, Basel 1972, S. 33.

125 Lonza AG (Hg.), 50 Jahre, S. 20.

126 H. J. Wörner, Unsere Lonza, in: Heimat am Hochrhein 1967/68, S. 115.

127 W. Maier, Die Lonza-Werke GmbH in Waldshut-Tiengen. Geschichte, Gegenwart und Zukunftsaspekte, in: Heimat am Hochrhein 1984, S. 35.

128 Fehr, Stickstofffrage, S. 143. 
die Grenzkontrollstelle mit nicht weniger als zwölf um den Leib geschnürten Würsten zu passieren versuchte. ${ }^{129}$

Die Lonza-Werke in Waldshut produzierten für die deutsche Kriegswirtschaft nicht nur Calciumcarbid, sondern verarbeiteten dieses ab 1916 auch zur Stickstoffverbindung Calciumcyanamid weiter. ${ }^{130}$ Die für diesen zusätzlichen Produktionsschritt erforderlichen Ausbauten wurden im Rahmen des bereits erwähnten Stickstoffprogramms des Reichsschatzamts von 1915 vorgenommen. ${ }^{131}$ Ursprünglich verpflichteten sich die Lonza-Werke gegenüber dem Reich zur „schleunigen Fertigstellung“ einer Anlage mit einer Kapazität von 40.000 Tonnen Kalkstickstoff pro Jahr, einem Wert, der wenig später auf 60.000 Jahrestonnen erhöht wurde. Der Staatssekretär des Reichsschatzamts bezeichnete „die baldige Errichtung der Anlagen im Interesse der Landesverteidigung und Volksernährung“als „dringend geboten“. Um den Ausbau der Fabrik möglichst schnell voranzutreiben wurden ,alle Lieferungen dafür als Kriegslieferungen angesehen und infolgedessen beschlagnahmte Stoffe (Metall, Öl, Gummi usw.) freigegeben“. ${ }^{132}$ Außerdem versprach das Reich den Lonza-Werken einen ,größeren Kredit“. ${ }^{133}$ Wie hoch die Bedeutung der Waldshuter Fabrik für die deutsche Stickstoffwirtschaft eingeschätzt wurde, macht eine Berechnung der Kriegschemikalien AG deutlich, die gegen Ende des Kriegs den Waldshuter Anteil an der deutschen Gesamterzeugung von Kalkstickstoff auf rund 15 Prozent schätzte. ${ }^{134}$

Da auch der in Waldshut produzierte Kalkstickstoff überwiegend zur Herstellung von Explosivstoffen und Munition genutzt wurde, betrachtete die Entente die Lonza-Werke folgerichtig als Teil der Kriegswirtschaft des militärischen Gegners, die es zu schwächen galt. Die Fabrikanlagen der Tochterfirma der schweizerischen Lonza AG wurden daher zu einem Angriffsziel des französischen Geheimdienstes. Als am 4. Mai 1917, also nur drei Tage nach dem misslungenen Anschlag auf das Werk der AIAG im schweizerischen Chippis, ein Anschlag auf das Waldshuter Lonza-Werk scheiterte, wurden die Täter auf der Flucht gefasst. Durch die anschließenden Befragungen und Untersuchungen konnten die

129 Wörner, Lonza, S. 115-116.

130 Meier, Lonza, S. 35.

131 Eucken, Stickstoffversorgung, S. 103.

132 BArch, R 4604 226, Schreiben des Staatssekretärs des Reichsschatzamts vom 25.02.1915; Fehr, Stickstofffrage, S. 144.

133 Waeser, Luftstickstoff-Industrie, S. 24.

134 BArch, R 8729 352, Leistungsfähigkeit und voraussichtliche Gesamterzeugung der Carbidwerke vom Oktober 1917 bis März 1918, einschließlich Kalkstickstoff-, Aceton- und Auslandsbedarf unter Voraussetzung der Materialbelieferug und Fertigstellung der Werke, abgeschlossen am 10.10.1917. 
Schweizer Behörden den „französischen Spionagedienst“ eindeutig als Drahtzieher identifizieren. ${ }^{335}$

Das Waldshuter Werk blieb nicht die einzige Fabrik, welche die Schweizer Lonza AG während des Ersten Weltkriegs auf deutschem Boden betrieb. Ein weiteres Werk nahm 1916 in Trattendorf in der Niederlausitz die Produktion von Calciumcarbid auf. ${ }^{136}$ In diesem Fall kamen allerdings weder Arbeitskräfte noch Ressourcen aus der weit über 500 Kilometer entfernten Schweiz zum Einsatz. Als Energielieferant war stattdessen das in unmittelbarer Nachbarschaft gelegene, seit 1915 im Bau befindliche Kohlekraftwerk der Niederlausitzer Kraftwerke AG vorgesehen, das über eine Schmalspurbahn Braunkohle aus der rund 4 Kilometer entfernten Grube „Brigitta“ beziehen sollte. Nach dreijähriger Bauzeit, während der auch zahlreiche Kriegsgefangene eingesetzt worden waren, lieferte das Großkraftwerk Trattendorf 1917 zum ersten Mal Elektrizität. ${ }^{137}$ Die Kriegschemikalien AG erachtete auch dieses Schweizer Werk auf deutschem Boden für wichtig genug, um bereits im Jahr der Eröffnung seine Erweiterung zu vereinbaren und dazu auch eine weitere finanzielle Unterstützung zu versprechen. Das von den Trattendorfer Lonza-Werken produzierte Calciumcarbid wurde unter anderem nach Waldshut und Trostberg geliefert und in den dortigen Werken zu Kalkstickstoff weiterverarbeitet, womit es ebenfalls der deutschen Stickstoffwirtschaft bzw. der deutschen Kriegswirtschaft zugutekam. ${ }^{138}$

\section{Zusammenfassung}

Dichtbesiedelte industrialisierte Staaten können sich nur bei hohen landwirtschaftlichen Flächenerträgen selbständig ernähren, wobei diese nur mit einer Zufuhr hoffremden Stickstoffdüngers erzielt werden können. Eine der wichtigsten Quellen gebundenen Stickstoffs war Ende des 19. bis Anfang des 20. Jahrhunderts

135 Schweizerischer Bundesrat, Bericht des Bundesrates über Begnadigungsgesuche (Wintersession 1919) vom 02.12.1919, in: Schweizerisches Bundesblatt 5, 1919, S. 670-673; BAR, E 21 14395, Urteil des Schweizerischen Bundesgerichts (Bundesstrafgericht) vom 11., 12. und 13. November 1918 in Sachen Schweizerische Bundesanwaltschaft gegen Zahnd Karl, Fricker Julius Arthur und Mougeot Maurice betreffend Sprengstoffverbrechen.

136 Lonza AG (Hg.), 75 Jahre, S. 33.

137 Lausitzer und Mitteldeutsche Bergbau-Verwaltungsgesellschaft mbH (Hg.), Kraftwerk Trattendorf, 1915-1996, Brieske 1996, S. 1-2.

138 BArch, R 8729 344, Vertrag zwischen der Kriegschemikalien Aktiengesellschaft und der Lonza-Werke Elektrochemische Fabriken GmbH vom 20.12.1916, S. 1-2. Siehe auch Fehr, Stickstofffrage, S. 146. 
der aus Südamerika importierte Chilesalpeter. Die Abhängigkeit von dieser aus Übersee eingeschifften, überlebenswichtigen Schlüsselressource veranlasste Experten unter dem Stichwort der Stickstofffrage bereits im 19. Jahrhundert zur Suche nach einer alternativen Stickstoffquelle, die sie nach der Jahrhundertwende in der Bindung des Stickstoffs der Luft fanden. Die Luftstickstofffixierung konnte zunächst durch das energieintensive Lichtbogen- und das Kalkstickstoff- sowie später durch das Hochdruckverfahren im industriellen Maßstab realisiert werden, wobei das Hochdruckverfahren das mit Abstand energieeffizienteste war.

Mit dem Beginn des Ersten Weltkriegs und der Verhängung der alliierten Blockade über die Mittelmächte wurde die im 19. Jahrhundert von Experten theoretisch erörterte Stickstofffrage für Deutschland auf einen Schlag zu einem akuten und existentiellen Problem. Dies nicht nur daher, weil mit der Einstellung der Chilesalpetereinfuhren plötzlich einer der wichtigsten hoffremden Stickstoffdünger wegfiel, sondern auch daher, weil Chilesalpeter ein unverzichtbarer Grundstoff für die militärisch bedeutsame Explosivstofferzeugung war und die Nachfrage nach Explosivstoffen kriegsbedingt stark anstieg. Die zeitgleich mit dem Einbruch des Angebots eintretende Vervielfachung der Nachfrage nach der Schlüsselressource Stickstoff veranlasste die deutsche Kriegswirtschaft zu weitreichenden Maßnahmen für den Ausbau der industriellen Fixierung des Stickstoffs der Luft. Dabei setzten die Behörden zunächst in erster Linie auf das Kalkstickstoffverfahren und lediglich in zweiter Linie auf das Hochdruckverfahren. Im Frühjahr 1916 änderte sich die Vorgehensweise insofern, als für die weiteren Ausbaupläne nur noch auf das wesentlich effizientere Hochdruckverfahren gesetzt wurde.

Insgesamt produzierte die deutsche Stickstoffwirtschaft in den ersten beiden Kriegsjahren trotz der ergriffenen Maßnahmen deutlich weniger Stickstoff als noch vor dem Krieg, weshalb zunächst weder der Ausfall des Chilesalpeters kompensiert, noch der kriegsbedingte Mehrbedarf gedeckt werden konnte. Erst ab 1915 wurde die Vorkriegsproduktion übertroffen. Bis 1918 erhöhte sich der jährliche Ausstoß auf nicht ganz das Doppelte der Vorkriegsproduktion. Da die Nachfrage aber ebenfalls stark zunahm, konnte sie während des gesamten Kriegs nie zu mehr als zwei Dritteln gedeckt werden. Eingespart wurde der fehlende Stickstoff primär in der Landwirtschaft, die daher während des gesamten Kriegs stark unterversorgt war.

Die Schweiz sah sich während des Ersten Weltkriegs ebenfalls mit einer schweren Störung des Außenhandels konfrontiert. Genau wie Deutschland konnte sie praktisch keinen Chilesalpeter mehr einführen. Da die Schweiz aber bereits über eine auf Wasserkraft basierende Kalkstickstoffindustrie verfügte, konnte zumindest der landwirtschaftliche Stickstoffbedarf während des ganzen Kriegs 
gedeckt werden. Daneben bestanden in der Schweiz zwei Lichtbogenanlagen, die sich ebenfalls die Wasserkraft in den Alpen zu Nutze machten und den Grundbedarf der Schweizer Armee nach Salpetersäure knapp decken konnten.

Aufgrund ihres eigenen Mangels an Chilesalpeter fiel die Schweiz als Transitland für eine Umgehung der alliierten Blockade außer Betracht. Diverse Schweizer Unternehmen belieferten die deutsche Kriegswirtschaft jedoch mit in der Schweiz selber, auf der Basis von Wasserkraft hergestellten Stickstoffverbindungen, darunter insbesondere Kalkstickstoff. Diese Ausfuhren waren aber derart bescheiden, dass sie nie mehr als 2 Prozent der deutschen Gesamterzeugung entsprachen. Wesentlich umfangreicher waren die Exporte von Calciumcarbid, einem energieintensiven Zwischenerzeugnis, welches die deutsche Stickstoffwirtschaft zur Herstellung von Kalkstickstoff benötigte, der dann wiederum zu Explosivstoffen und Munition weiterverarbeitet wurde. Schweizer Unternehmen führten während des Kriegs rund 200.000 Tonnen Karbid nach Deutschland aus, womit geschätzte 30 Prozent der gesamten deutschen Kalkstickstofferzeugung zwischen 1914 und 1918 hätten alimentiert werden können. Welcher Anteil des Schweizer Karbids aber tatsächlich der deutschen Stickstoffwirtschaft zugeführt wurde, lässt sich nicht mehr im Detail rekonstruieren.

Während es sich bei Calciumcarbid faktisch um einen Speicher von Schweizer Wasserkraft handelte, exportierten schweizerische Elektrizitätsunternehmen auch direkt Elektrizität nach Deutschland, wo sie der Stickstoffwirtschaft zugeführt wurde. Zu den Beziehern zählte die „Lonza-Werke Elektrochemische Fabriken $\mathrm{GmbH}^{\prime}$, eine auf der deutschen Seite des Rheins gelegene Tochterfirma der schweizerischen Lonza AG. Diese produzierte ab 1915 zunächst Calciumcarbid sowie ab dem Folgejahr Kalkstickstoff und war somit Teil der deutschen Stickstoffwirtschaft. Indem sie nicht nur Schweizer Strom, sondern auch Schweizer Kalk bezog und weitgehend mit Schweizer Arbeitskräften betrieben wurde, die überdies auf der Schweizer Seite des Rheins verpflegt wurden, zog sie für ihre Produktion so weit wie nur möglich Schweizer Ressourcen heran. Damit wurde das Schweizer Stickstoffwerk auf deutschem Boden auch zu einer Zielscheibe französischer Agenten, deren Versuch zur Zerstörung des Werks 1917 allerdings scheiterte. Neben den Waldshuter Werken gründete die Lonza AG 1915 in Trattendorf ein weiteres Schweizer Werk auf deutschem Boden, das auf der Basis von Kohle ebenfalls Calciumcarbid für die deutsche Stickstoffwirtschaft produzierte. 


\section{Bionote}

\section{Sandro Fehr}

studierte Geschichte und Politikwissenschaft. Anschließend war er am Historischen Institut der Universität Bern tätig, wo er 2012 mit einer verkehrshistorischen Arbeit über die Luftfahrtinfrastruktur in der Schweiz promovierte. Die Kriegswirtschaft während des Ersten Weltkriegs bildet einen weiteren Schwerpunkt seiner Forschungstätigkeit. Seit 2013 arbeitet er hauptberuflich als Gymnasiallehrer an der Kantonsschule Rychenberg in Winterthur. Bücher: S. Fehr, Die Stickstofffrage in der deutschen Kriegswirtschaft des Ersten Weltkriegs und die Rolle der neutralen Schweiz, Nordhausen 2009; S. Fehr, Die Erschliessung der dritten Dimension. Entstehung und Entwicklung der zivilen Luftfahrtinfrastruktur in der Schweiz, 1919-1990, Zürich 2014. 
- 10.1515/jbwg-2015-0019

Downloaded from PubFactory at 09/07/2016 04:10:57PM via Universitätsbibliothek Bern 